SBI-IFUSP

$305 \mathrm{M} 810 \mathrm{~T} 3642$

UNIVERSIDADE DE SÃO PAULO

Instituto de Física

\title{
Entropia e Holografia em Teorias da Gravitação
}

\section{Luis Alejandro Correa Borbonet}

Orientador:

Prof. Dr. Elcio Abdalla

Tese apresentada ao Instituto de Física da Universidade de São Paulo para obtenção do título de Doutor em Ciências.
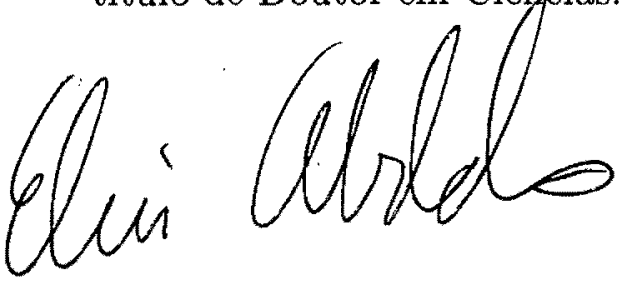

Comissão Examinadora:

Prof. Dr. Elcio Abdalla (orientador)

Prof. Dr. Victor de Oliveira Rivelles (IFUSP)

Prof. Dr. Frank Michael Forger (IME/USP)

Prof. Dr. Alberto Vazquez Saa (UNICAMP)

Prof. Dr. Ilia Shapiro (UFJF)

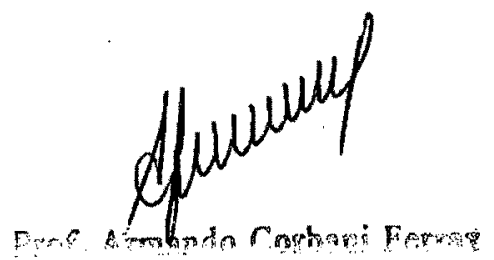

São Paủlo

2002

INSTITUTO DE FISICA

Serviço de Ribliotesa e Informação

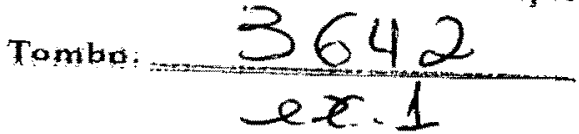


523.1

C824e

D.x.1

FICHA CATALOGRÁFICA

Preparada pelo Serviço de Biblioteca e Informação do Instituto de Física da Universidade de São Paulo

Correa Borbonet, Luis Alejandro

Entropia e Holografia em Teorias da Gravitação.

São Paulo 2002.

Tese (Doutoramento) - Universidade de São Paulo Instituto de Física - Departamento de Física Matemática

Orientador: Prof. Dr. Élcio Abdalla

Área de Concentração: Física

Unitermos: 1. Princípio Holográfico;

2. Teorias de Gravitação;

3. Entropia. 


\section{Resumo}

Estudamos a entropia em várias situações na gravidade, verificando se seu comportamento é holográfico, obedecendo à lei de área de Bekenstein. Inicialmente, usando o método da "parede de tijolos", calculamos, em diversos casos, a entropia estatística de um campo escalar num fundo não trivial. Tal fundo é gerado por buracos negros de 4 ou 5 dimensões com cargas. A fórmula da entropia de Bekenstein é geralmente satisfeita, mas algumas correções são discutidas no caso pentadimensional. Este método é também aplicado para soluções tipo buracos negros na gravidade de Lovelock. Resulta que o método de "parede de tijolos", apesar de correto para a teoria de Einstein-Hilbert, pode não ser válido em geral, o que também acontece com a lei de área. Algumas propriedades concernentes à teoria de cordas, especialmente a tecnologia das $\mathrm{D}$-branas, são revistas naqueles aspectos necessários para este trabalho. Também estudamos e calculamos o limite superior da entropia para a gravidade de Lovelock. Finalmente, verificamos a validade do princípio holográfico num universo de $(4+n)$ dimensões numa fase inflacionária assimétrica. 


\begin{abstract}
We study the entropy for various situations in gravity, checking whether its behavior is holographic, obeying Bekenstein's area law. First, using the brick wall method, we compute the statistical entropy of a scalar field in a nontrivial background in different cases. Such a background is generated by four and five dimensional black holes with charges. The Bekenstein entropy formula is generally obeyed, but corrections are discussed in the latter case. This method is applied also to the black hole solutions of the Lovelock gravity. It turns out that the brickwall method, though correct for the EinsteinHilbert theory, may fail in general. The same happens to the area law. Some properties concerning string theory, especially the D-branes technology, are reviewed while necessary to this work. Furthermore, we study and compute the upper limit of the entropy for the Lovelock gravity. Finally, we check the validity of the holographic principle in a $(4+n)$ dimensional universe in an asymmetric inflationary phase.
\end{abstract}


Dedico aos meus pais Angelica e Luis.

"Puesto que se tiene la dote de la razón, se tiene él deber de usarla." Jose Marti. 


\section{Agradecimentos}

- Agradeço a meu orientador Prof. Dr. Elcio Abdalla pela condução da pesquisa e pela troca livre de ideias, e pela ajuda em diversos momentos.

- Agradeço ao povo do Brasil pela acolhida e hospitalidade.

- Agradeço à Capes pelo apoio financeiro que possibilitou a realização do Doutorado.

- Agradeço aos colegas do IFUSP e IFT pelo ambiente cordial e ayuda em diversos momentos. Entre eles Marcelo, Bertha, Carlos, Leticia, Pablo, Osada, Leonardo, Arnaldo, Sé, Karlucio, Francisco, Alexandre, Alessandro, Roberto, Cristiane.

- Aos amigos Jorgito, Paulo, Erica, Elisa, Crispino pelos bons momentos e a amizade.

- Agradeço aos meus pais pelo apoio durante todos estes anos. Também à familia e os amigos em Cuba.

- Finalmente, a Iri e Daniele Alejandro pela companhia magnífica e os bons momentos vividos estes anos. 


\section{Sumário}

1 Introdução

2 Entropia do Buraco Negro. 12

2.1 Modelo da Parede de Tijolos. . . . . . . . . . . . . 12

2.1.1 Buracos Negros em $D=4$ e $D=5$ com cargas. . . . . 18

2.1.2 Método da Parede de Tijolos na gravidade de ordem superior. . . . . . . . . . . . . . . 23

2.2 Entropia e Teoria Conforme. . . . . . . . . . . . . . . 31

2.2.1 Algebra de Virasoro e a Fórmula de Cardy. . . . . . . 32

2.2.2 Teoria conforme na Relatividade Geral. . . . . . . . . 36

2.2.3 Configuração do modo zero e contagem dos estados . . 39

2.3 Método das D-branas . . . . . . . . . . . . . . . . 40

2.3.1 Solução de brana-negra na teoria de supercorda Tipo IIB . . . . . . . . . . . . . . . . 44

2.3.2 Número de estados microscópicos. . . . . . . . . . . 45

3 Limites para a Entropia. $\quad \mathbf{4 7}$

3.1 Relação $S / E \ldots \ldots \ldots \ldots \ldots \ldots$. . . . . . . . . 47

3.1.1 Relação S/E na gravidade de ordem superior . . . . . . 49

3.2 Limites holográficos da entropia na cosmologia. . . . . . . . 51

3.2 .1 Universos Chatos. . . . . . . . . . . . . . 51

3.2 .2 Universo Fechado e Inflação. . . . . . . . . . . . . . . 53

3.3 Exemplo . . . . . . . . . . . . . . . . . 5 53

3.4 Fórmula de Cardy-Verlinde . . . . . . . . . 68

4 Conclusões $\quad 70$

A Entropia de Gibbons-Hawking $\quad 72$ 
B Formalismo Hamiltoniano para a ação de Lovelock.

$\begin{array}{ll}\text { C Constantes } & 76\end{array}$

D Supercordas e D-branas $\quad \mathbf{7 7}$

D.1 Supercordas . . . . . . . . . . . . . . . 77

D.2 D-branas . . . . . . . . . . . . . . . . . . 79 


\section{Capítulo 1}

\section{Introdução}

Em nossa visão do mundo físico, está firmemente estabelecida a idéia de que os graus de liberdade estão espalhados pelos campos que moram no espaço tridimensional. Os estudos da gravidade quântica têm conduzido muitos físicos teóricos a acreditar que esta visão implica em uma superestimativa dos graus de liberdade, e para que o estudo da gravidade a nível quântico tenha sentido, é necessário colocar um pequeno "corte". De acordo com esta filosofia nosso mundo é tão rico em estrutura quanto uma teoria em uma rede tridimensional com um espaço mínimo da ordem da escala de Planck. Mas, em 1993, G.'t Hooft [1] propôs uma imagem ainda mais radical da natureza. Ele sugeriu que para se ter uma descrição completa da natureza bastava conhecer as características da rede bidimensional na fronteira de nosso mundo. Dois anos depois, L. Susskind apresentou as mesmas idéias de um modo mais claro [2]. Atualmente esta idéia é conhecida como "Princípio Holográfico".

A principal motivação para o surgimento deste Princípio foi o conjunto de trabalhos sobre a termodinâmica dos buracos negros feito por Bekenstein [3]. Ele argumentou que a entropia de um buraco negro tinha que ser proporcional à sua área. A descoberta da radiação de Hawking [4] alguns anos depois permitiu estabelecer de modo preciso o fator de proporcionalidade, $1 / 4$, entre a entropia de um buraco negro e sua área. Deste modo, foi estabelecida a bem conhecida fórmula de Bekenstein-Hawking

$$
S_{B H}=\frac{A}{4 G} .
$$

Mas a idéia de 't Hooft e Susskind foi fortalecida depois de ser apresentada a correspondência AdS/CFT [5] no contexto da teoria de cordas. No 
trabalho de Maldacena conjetura-se a existência de uma dualidade entre a teoria de supercordas ou da supergravidade em espaços $A d S_{d+1}$ (Anti-de Sitter), e teorias de gauge conformes morando na borda, em $d$ dimensões. Nos trabalhos [6,7] a proposta de Maldacena foi colocada em bases firmes e foi estabelecida a forma precisa através da qual uma teoria deve ser mapeada na outra. A prescrição estabelece que o limite assintótico, quando os campos se aproximam da borda, da função de partição da teoria no volume é o funcional gerador para as funções de correlação da teoria conforme na borda. Portanto $[8]$,

$$
\left\langle e^{\int d^{d} x \phi_{0}(x) \mathcal{O}(x)}\right\rangle_{T C C}=\mathcal{Z}_{\text {corda }}\left[\left.\phi(x, z)\right|_{z=0}=\phi_{0}(x)\right],
$$

onde $\phi_{0}$ é o valor do campo na fronteira do espaço AdS.

Neste contexto a expressão (1.2) é uma manifestação muito explícita da holografia, toda a dinâmica da física no volume pode ser encontrada a partir das funções de correlação na fronteira. Deste modo, a correspondência AdS/CFT é um bom laboratório para o entendimento do princípio holográfico.

Mas nosso interesse é nos aprofundarmos na outra exigência do Princípio Holográfico, que estabelece que o número de graus de liberdade por unidade de área não seja superior a 1 bit por área de Planck (Fig.1), e com esse objetivo apresentamos nesta tese vários modos de calcular a entropia, ou seu limite superior.

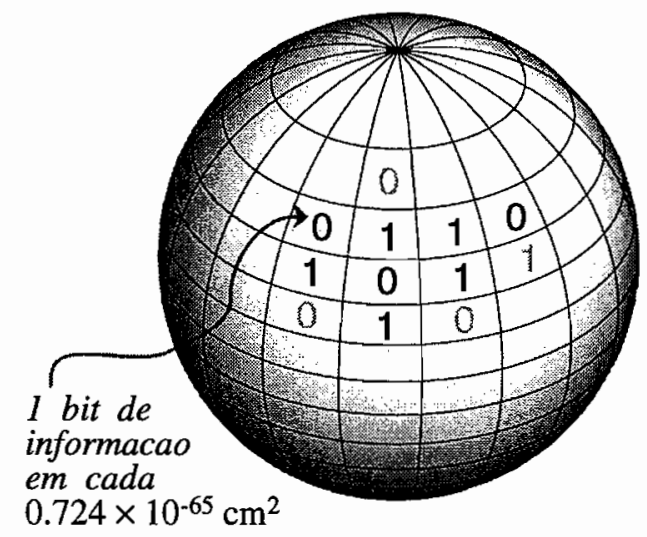

Figura 1. Informação no horizonte do buraco negro.

Apesar da termodinâmica dos buracos negros estar bem estabelecida, a fundamentação estatística ainda é um mistério. Nos últimos anos, alguns 
progressos parciais foram conseguidos por teorias tão diversas como a teoria de cordas [51] e a "geometria quântica" [11], mas isto acrescentou um problema: temos várias descrições microscópicas concorrentes de um mesmo fenômeno sem um caminho claro para entender por que chegam a resultados idênticos. Talvez a direção mais plausível para solucionar esta questão seja a sugerida por Strominger [10] recentemente. Independentemente dos detalhes dos graus de liberdade, qualquer teoria quântica da gravidade tem um grupo de simetria particular conhecido como o grupo dos difeomorfismos, que realmente vem da teoria clássica que é a Relatividade Geral. Então é esperado que a estrutura clássica do grupo dos difeomorfismos seja suficiente para governar o comportamento da densidade de estados quântica. Com efeito, o objetivo é encontrar os graus de liberdade microscópicos compatíveis com a estrutura macroscópica do buraco negro. Através da fórmula de Boltzmann

$$
S=k_{B} \ln N \text {, }
$$

encontra-se a entropia e depois confere-se sua concordância com a fórmula de Bekenstein-Hawking.

Nesta tese mostraremos três modos de calcular a entropia do buraco negro. Primeiramente mostraremos o método proposto por 't Hooft [13] onde ele identifica a entropia do buraco negro com a entropia estatística de um gas térmico de partículas quânticas com uma condição de fronteira (tipo parede) na vizinhança do horizonte de eventos do buraco negro. Devido a este fato o método é conhecido como "brick wall", parede de tijolos. No trabalho original foi considerado o caso mais simples de um campo escalar propagando-se perto de um buraco tipo Schwarzschild com massa M. Depois de cálculos relativamente simples 't Hooft encontrou que a entropia era proporcional à área. Mas também encontrou que $S$ era proporcional a $\alpha^{-2}$ sendo $\alpha$ a distância própria do horizonte à parede. Portanto o cálculo apresenta uma divergência quadrática no horizonte. A natureza desta divergência pode ser entendida em termos do desvio infinito para o vermelho, entre o horizonte e o infinito físico. Para comprender isto Susskind e Uglum [24] sugeriram que a divergência podia ser absorvida na renormalização da constante de Newton, e portanto o cálculo feito por 't Hooft podia ser interpretado como a correção de um loop à fórmula "clássica" de Bekenstein-Hawking. Com o objetivo de testar este método estudamos os campos escalares em outros tipos de buracos negros, seja com cargas ou em dimensões maiores, neste caso em cinco dimensões. Também com o objetivo de conferir a ideia de 't Hooft em situações mais gerais aplicamos o método em buracos negros que 
são soluções da gravidade de Lovelock[25], que resulta interessante por ser uma extensão da Relatividade Geral.

Posteriormente, apresentamos o método de Solodukhin[45] que faz uso das ferramentas da teoria de campos conforme (TCC) para encontrar a entropia do buraco negro. Motivado por trabalhos anteriores onde foi proposto que o horizonte tem uma natureza conforme, Solodukhin mostrou que depois de reduzir dimensionalmente a ação de Einstein-Hilbert na vizinhança do horizonte de eventos, obtém-se um teoria conforme (Liouville). Depois, calculando a álgebra de Virasoro para esta teoria e conseqüentemente determinando a carga central $c$ e o autovalor $L_{0}$ do operador de Virasoro, foi possível encontrar a entropia com a ajuda da fórmula de Cardy.

A teoria de cordas, no ano de 1996 conseguiu um feito significativo depois que Vafa e Strominger [51] calcularam a entropia de um buraco negro pentadimensional, obtido a partir de 10 dimensões mediante uma compactificação de $K_{3} \times S^{1}$. Na tese estudaremos um buraco negro mais simples, também pentadimensional, mas obtido por compactificação toroidal. Neste caso o buraco negro e a entropia são obtidos usando a chamada tecnologia das D-branas. Na teoria de cordas a contagem dos estados que levam à entropia do buraco negro é feita no limite da constante de acoplamento fraca. Neste limite os estados são as flutuações quânticas ao redor das D-branas que moram no espaço plano. Aparentemente esta contagem não tem relação com o problema da entropia do buraco negro. Mas para entender isto deve-se levar em conta o caráter especial das D-branas e o fato de que estamos trabalhando com uma teoria supersimétrica. Dentro do contexto das dualidades na teoria de cordas ficou claro que a aparição das D-branas era necessária devido ao fato de que essas dualidades trocam os estados Neveu-Schwartz Neveu-Schwartz e Ramond-Ramond, e os estados de teoria de cordas perturbativa só portam cargas NS. As D-branas portam as cargas do setor Ramond e portanto permitem a realização das dualidades entre os estados.

Uma vantagem de se estar trabalhando com uma teoria supersimétrica é a existência dos estados BPS. Estes estados têm a propriedade de ser invariantes perante a ação de alguns dos geradores supersimétricos. O número dos estados BPS é essencialmente um invariante topológico e portanto não muda se a constante de acoplamento mudar continuamente. Usando este fato a contagem dos estados pode ser feita, seja no regime forte ou no regime fraco da constante de acoplamento. Os estados do buraco negro em que estamos interessados estão no regime forte, mas fazer a contagem é mais fácil no regime fraco. Então o cenário é o seguinte: se inicia a contagem dos estados BPS 
no regime fraco e depois se incrementa a constante de acoplamento de cordas $g$. Devido ao fato de que a constante de Newton cresce com $g^{2}$ o campo gravitacional fica mais forte e se chega a um ponto onde temos a formação de um buraco negro. Conseqüentemente, o número dos estados BPS, que tem as mesmas cargas que o buraco negro, é identificado com a exponencial da entropia do buraco negro.

Outro interesse nosso é estudar em diversas situações os limites superiores da entropia. No ano 1981 Bekenstein propôs que a relação entre a entropia e a energia está limitada pela dimensão do corpo ou seja $S / E \leq 2 \pi R$. Isto foi demostrado para o caso onde os efeitos gravitacionais são máximos, os buracos negros, e também para sistemas com autogravidade desprezível. Na teses testamos como se comporta esta relação no caso da gravidade de Lovelock [25].

A idéia de 't Hooft e a correspondência AdS/CFT induziram Fischler e Susskind a tentar estender o Princípio Holográfico à Cosmologia. No trabalho [64] eles propuseram um critério para calcular a grandeza $S / A$ nos modelos cosmológicos. A idéia é que o fluxo de entropia através de um frente de onda de luz contraindo-se seja menor ou igual que a área da superfície onde a onda de luz foi originada. Na tese aplicaremos este critério a um modelo cosmológico [68] encontrado dentro do contexto das teorias com dimensões extras. Este modelo, proposto por Arkani-Hamed et al é interessante porque apresenta uma alternativa ao processo de reaquecimento presente nos modelos inflacionários padrões.

$\mathrm{Na}$ última seção da tese damos uma breve descrição de uma idéia recente proposta por E. Verlinde [77]. Nesse trabalho ele mostra, com determinadas condições, que é possível mapear a fórmula de Friedmann na fórmula de Cardy.

A tese é organizada como segue. No Capítulo 2 se calcula a entropia dos buracos negros usando três métodos diferentes. O primeiro a ser apresentado é o de 't Hooft. Como continuação é mostrado o método de Solodukhin que usa as ferramentas básicas das teorias conformes. Finalmente estudamos o formalismo de cordas e D-branas para a contagem dos graus de liberdade do buraco negro. No Capítulo 3 estudamos os limites da entropia. Primeiramente o límite de Bekenstein na gravidade de Lovelock e depois o critério FS para o modelo cosmológico de Arkani-Hamed et al. As conclusões são apresentadas no Capítulo 4. No Apêndice A mostramos o cálculo da fórmula da entropia de Bekenstein-Hawking pelo mesmo Hawking e por Gibbons. No Apêndice B é apresentada a forma de calcular a ação de Lovelock. No 
Apêndice $\mathrm{C}$ mostramos as constantes obtidas no cálculo da energia livre da seção 2.1.2. No último Apêndice se colocam elementos básicos da teoria de cordas e D-branas. 


\section{Capítulo 2}

\section{Entropia do Buraco Negro.}

Um tema central na investigação da gravidade quântica nos últimos anos tem sido o da entropia dos buracos negros. Este problema já foi discutido a partir de diversas perspectivas [12], e o desafio é derivar a fórmula de BekensteinHawking a partir de primeiros princípios. Neste capítulo mostraremos três modos que tem sido propostos para explicar este fato.

\subsection{Modelo da Parede de Tijolos.}

No trabalho [13], 't Hooft tentou formular uma explicação microfísica da entropia do buraco negro, considerando os modos de um campo escalar na sua vizinhança. No cálculo encontra-se uma divergência no número de modos devido ao deslocamento infinito para o ultra-violeta no horizonte de eventos. Para regularizar este cálculo 't Hooft introduziu um corte ultravioleta que chamou de "brick wall", parede de tijolos, impondo a anulação do campo escalar para uma distância próxima ao horizonte. Além de encontrar, como era de se esperar, as grandezas termodinâmicas que descrevem os campos no espaço quase-chato, proporcionais ao volume, ele também encontrou contribuções da parede proporcionais à área. Contudo, essas contribuções eram proporcionais a $\alpha^{-2}$, onde $\alpha$ e a altitude própria da parede sobre o horizonte e portanto divergente para o limite $\alpha \rightarrow 0$. Para uma escolha determinada de $\alpha$, 't Hooft conseguiu reproduzir a fórmula de Bekenstein-Hawking.

Mostraremos agora o método para o caso mais simples, um buraco negro estático e sem carga, com massa $\mathrm{M}$, que é descrito pelo elemento de linha de 
Schwarzchild,

$$
d s^{2}=g_{\mu \nu} d x^{\mu} d x^{\nu}=-h(r) d t^{2}+h(r)^{-1} d r^{2}+r^{2}\left(d \theta^{2}+\sin ^{2} \theta d \varphi^{2}\right),
$$

onde $h(r)=1-r_{0} / r$ e $r_{0}=2 M$.

Consideremos nesta geometria um campo escalar massivo livre, cuja ação é dada por

$$
S=\int d^{4} x \mathcal{L}
$$

onde

$$
\mathcal{L}=-\frac{\sqrt{-g}}{2}\left(\partial^{\mu} \Phi \partial_{\mu} \Phi+m^{2} \Phi^{2}\right)
$$

é a densidade de lagrangeana, e $g \equiv \operatorname{det}\left(g_{\mu \nu}\right)$ é o determinante da métrica.

Então a equação dinâmica obtida pelo princípio da mínima ação é a equação de Klein-Gordon [21]

$$
\frac{1}{\sqrt{-g}} \partial_{\mu}\left(\sqrt{-g} g^{\mu \nu} \partial_{\nu} \Phi\right)-m^{2} \Phi=0
$$

O método de 't Hooft consiste em introduzir um corte perto do horizonte de eventos, de forma que a condição de fronteira

$$
\Phi=0 \quad \text { para } \quad r \leq r_{0}+\epsilon
$$

seja satisfeita.

Para eliminar as divergências infravermelhas outro corte é introduzido para distâncias longe do horizonte, $L \gg r_{0}$,

$$
\Phi=0 \quad \text { para } \quad r \geq L .
$$

Em um espaço-tempo estático e esfericamente simétrico, como é o caso do espaço-tempo de Schwarzschild, a função $\Phi$ pode ser escrita na forma

$$
\Phi(t, r, \theta, \varphi)=e^{-i E t} R(r) Y(\theta, \varphi) .
$$

Usando (2.7) e a equação obedecida por $Y(\theta, \psi)$

$$
\triangle Y(\theta, \varphi)=-l(l+1) Y(\theta, \varphi),
$$

obtemos a partir de (2.4) a equação diferencial a ser satisfeita pela função radial $R(r)$, a saber

$$
h^{-1} E^{2} R(r)+\frac{1}{r^{2}} \partial_{r}\left[r^{2} h \partial_{r} R(r)\right]-\frac{l(l+1)}{r^{2}} R(r)-m^{2} R(r)=0
$$


Usando a aproximacão $\mathrm{WKB}^{1}$ consideramos $R(r)=\rho(r) e^{i S(r)}$, onde a função $\rho(r)$ é uma amplitude que varia suavemente e $S(r)$ é uma fase que varia rapidamente. $\mathrm{Na}$ aproximação de primeira ordem a contribuição da primeira derivada de $S$ é a mais importante. Desta forma, a partir da equação (2.9) obtemos o número de onda radial $K \equiv \partial_{r} S$, dado por

$$
K=\left(1-\frac{r_{0}}{r}\right)^{-1} \sqrt{E^{2}-\left(1-\frac{r_{0}}{r}\right)\left(\frac{l(l+1)}{r^{2}}+m^{2}\right)},
$$

de onde segue que o número de modos radiais $n_{r}$ é dado por

$$
\pi n_{r}=\int_{r_{0}+\epsilon}^{L} d r K(r, l, E) .
$$

Com o objetivo de calcular a entropia do sistema, primeiramente calculamos a energia livre $F$ de um banho térmico de partículas escalares com a temperatura $1 / \beta$,

$$
e^{-\beta F}=\sum e^{-\beta E_{N_{\tau}}},
$$

onde $E_{N_{\tau}}$ é a energia total correspondente ao estado quântico $\tau$. A soma também inclui as degenerescências dos estados quânticos, portanto temos

$$
e^{-\beta F}=\prod_{\left(n_{r}, l, m_{l}\right)} \frac{1}{1-\exp (-\beta E)},
$$

onde $\left(n_{r}, l, m_{l}\right)$ é o conjunto de números quânticos associados a este problema. $O$ produto $\prod$ leva em conta a contribuição de todos os modos. O fator $\left(1-e^{-\beta E}\right)^{-1}$ é devido ao fato de que estamos tratando com bósons, para os quais o número de ocupação pode tomar valores em todos os inteiros não negativos, de modo que obtemos a soma geométrica

$$
\sum_{n=0}^{\infty} e^{-\beta n E}=\frac{1}{1-\exp (-\beta E)} .
$$

Levando em conta as equações anteriores, a energia livre pode ser escrita como

$$
\begin{aligned}
F & =\frac{1}{\beta} \sum_{n_{r}, l, m_{l}} \ln \left(1-e^{-\beta E}\right) \\
& =\frac{1}{\beta} \int d l(2 l+1) \int d n_{r} \ln \left(1-e^{-\beta E}\right),
\end{aligned}
$$

\footnotetext{
${ }^{1}$ Neste sentido o cálculo é semi-clássico
} 
onde aproximamos as somas por integrais, o que corresponde a uma aproximação semi-clássica.

Integrando por partes e usando (2.11) obtemos

$$
\begin{aligned}
F= & -\int d l(2 l+1) \int d E \frac{1}{\exp (\beta E)-1} n_{r} \\
= & -\frac{1}{\pi} \int d l(2 l+1) \int d E \frac{1}{\exp (\beta E)-1} \int_{r_{0}+\epsilon}^{L} d r \\
& \times\left(1-\frac{r_{0}}{r}\right)^{-1} \sqrt{E^{2}-\left(1-\frac{r_{0}}{r}\right)\left(\frac{l(l+1)}{r^{2}}+m^{2}\right)} .
\end{aligned}
$$

A integração na variável $l$ pode ser feita explicitamente e somente sobre aqueles valores para os quais a raiz quadrada exista,

$$
\begin{gathered}
\int d l(2 l+1)\left[E^{2}-\left(1-\frac{r_{0}}{r}\right)\left(\frac{l(l+1)}{r^{2}}+m^{2}\right)\right]^{\frac{1}{2}}= \\
\frac{2}{3} r^{2}\left(1-\frac{r_{0}}{r}\right)^{-1}\left(E^{2}-\left(1-\frac{r_{0}}{r}\right) m^{2}\right)^{\frac{3}{2}},
\end{gathered}
$$

de onde segue que

$$
F=-\frac{2}{3 \pi} \int d E \frac{1}{\exp (\beta E)-1} \int_{r_{0}+\epsilon}^{L} d r r^{2}\left(1-\frac{r_{0}}{r}\right)^{-2}\left[E^{2}-\left(1-\frac{r_{0}}{r}\right) m^{2}\right]^{3 / 2}
$$

Note-se que o integrando diverge no horizonte de eventos. Primeiramente introduzimos a variável $y=r / r_{0}$. Substituindo na equação anterior obtemos

$$
F=-\frac{2 r_{0}^{3}}{3 \pi} \int d E \frac{1}{\exp (\beta E)-1} \int_{1+\bar{\epsilon}}^{\bar{L}} d y y^{2}\left(1-\frac{1}{y}\right)^{-2}\left[E^{2}-\left(1-\frac{1}{y}\right) m^{2}\right]^{3 / 2}
$$

onde $\bar{\epsilon}=\frac{\epsilon}{r_{0}}, \bar{L}=\frac{L}{r_{0}}$. A contribuição a esta integral para valores grandes de $y$ é

$$
F_{L}=-\frac{2 L^{3}}{9 \pi} \int_{m}^{\infty} d E \frac{\left(E^{2}-m^{2}\right)^{3 / 2}}{\exp (\beta E)-1}
$$

Podemos reconhecer que este é o termo usual que vem do vazio que rodeia o sistema. 
Vizinho ao horizonte, para $y$ perto de 1 , temos

$$
F_{\epsilon}=-\frac{2 r_{0}^{3}}{3 \pi} \int_{0}^{\infty} d E \frac{E^{3}}{\exp (\beta E)-1} \int_{1+\bar{\epsilon}}^{\bar{L}} d y(y-1)^{-2} .
$$

Depois, usamos a conhecida fórmula

$$
\int_{0}^{\infty} d E \frac{E^{3}}{e^{\beta E}-1}=\frac{\pi^{4}}{15 \beta^{4}},
$$

e integramos em $y$. A expressão para $F_{\epsilon}$ se reduz a

$$
F_{\epsilon}=-\frac{2 \pi^{3}}{45 \epsilon} \frac{r_{0}^{4}}{\beta^{4}}
$$

permitindo-nos calcular diretamente a entropia a partir da expressão

$$
S_{\epsilon}=\beta^{2} \frac{\partial F_{\epsilon}}{\partial \beta}=\frac{8 \pi^{3}}{45 \epsilon} \frac{r_{0}^{4}}{\beta^{3}} .
$$

Agora só precisamos calcular a temperatura $1 / \beta$. A temperatura de Hawking do buraco negro é definida como

$$
T_{H}=\beta_{H}^{-1}=\frac{\kappa_{s}}{2 \pi},
$$

onde $\kappa_{s}$ e a "surface gravity", ou seja, a aceleração da gravidade na superfície do buraco negro, correspondente ao raio de Schwarzschild. Esta é expressa em termos da métrica como

$$
\kappa_{s}=\left[\sqrt{g^{r r}}\left(\partial_{r} \sqrt{-g_{t t}}\right)\right]_{r=r_{0}} .
$$

Para a métrica (2.37) obtemos

$$
\kappa_{s}=\frac{1}{2 r_{0}} .
$$

O inverso da temperatura de Hawking fica

$$
\beta_{H}=4 \pi r_{0},
$$

e a entropia

$$
S=\frac{r_{0}}{360 \epsilon}
$$


Esta expressão pode ser transformada usando a "distância invariante"

$$
\alpha=\int d s=\int_{r_{0}}^{r_{0}+\epsilon} d r \frac{1}{\sqrt{1-r_{0} / r}}=\sqrt{4 \epsilon r_{0}},
$$

Reescrevendo a entropia em termos da distância invariante obtemos

$$
S=\frac{r_{0}}{360 \epsilon}=\frac{A}{1440 \pi \epsilon r_{0}}=\frac{A}{360 \pi\left(\int d s\right)^{2}},
$$

onde $A=4 \pi r_{0}^{2}$ é a área do horizonte.

Então, a entropia do campo escalar na vizinhança do buraco negro tem uma divergência quadrática na distância própria acima do horizonte. Susskind e Uglum [24] sugeriram que esta divergência tem a forma apropriada para ser absorvida na fórmula de Bekenstein-Hawking mas com a constante da gravitação de Newton renormalizada. Portanto o cálculo feito pode ser considerado como uma correção de um loop à entropia "clássica" calculada por Gibbons e Hawking [56] (ver Apêndice A), ou seja,

$$
S=\frac{A}{4 G_{R}},
$$

onde $G_{R}$ é a constante de Newton renormalizada e satisfaz à relação

$$
\frac{1}{4 G_{R}}=\frac{1}{4 G_{N}}+\frac{1}{360 \pi \alpha^{2}}
$$

Isto fica evidente nos cálculos da ação efetiva para a métrica quando se considera o campo escalar (2.3). A ação efetiva para a métrica se obtém quando nas integrais de caminho o campo escalar é integrado [21],[20]. Neste caso a integração é Gaussiana, levando-nos à raiz do determinante do propagador; a contribuição para a ação efetiva gravitacional, que é o logaritmo deste resultado, é $W(g)=-\frac{i}{2} \operatorname{Tr} \log \left[-G_{F}\left(g, m^{2}\right)\right]$. Esta expressão é divergente e deve ser regularizada para estar definida apropriadamente. As divergências desta ação efetiva de um loop, assim como sua dependência na métrica, são identificadas facilmente usando a expansão adiabática para a representação de tempo próprio de DeWitt-Schwinger do propagador. Isto nos leva à representação da ação de um loop do campo escalar como uma serie assintótica ${ }^{2}$ :

$$
W(g)=-\frac{1}{32 \pi^{2}} \int d^{4} x \sqrt{-g} \int_{0}^{\infty} \frac{d s}{s^{3}} \sum a_{n}(x)(i s)^{n} e^{-i m^{2} s},
$$

\footnotetext{
${ }^{2}$ Neste caso o determinante de Van Vleck é igual a um [21].
} 
onde os coeficientes $a_{n}(x)$ são funcionais da geometria local em $x$. Portanto são expressões locais construídas em termos da métrica e do tensor de curvatura. Por exemplo os coeficientes dos primeiros três termos são

$$
\begin{aligned}
& a_{0}(x)=1 \\
& a_{1}(x)=\frac{1}{6} R, \\
& a_{2}(x)=\frac{1}{180} R^{a b c d} R_{a b c d}-\frac{1}{180} R^{a b} R_{a b}+\frac{1}{30} R+\frac{1}{72} R^{2} .
\end{aligned}
$$

No presente caso em quatro dimensões, as divergências ultra-violeta aparecem quando $s \rightarrow 0$ nos três primeiros termos da serie. Um possível método para regularizar esta ação é a regularização de Pauli-Villars. Mas nosso único interesse é mostrar concisamente que a divergencia encontrada na entropia está vinculada à renormalização da constante de Newton. Para mais detalles ver [20].

Considerando a expansão (2.34), o termo linear da ação gravitatoria fica

$$
\begin{aligned}
I_{e f} & =I_{g}+W(g) \\
& =\int d^{4} x \sqrt{-g} R\left(\frac{1}{16 \pi G_{N}}+\frac{B}{12 \pi}\right) .
\end{aligned}
$$

onde B é um termo divergente[21].

Isto mostra que o problema da divergência da entropia está intrinsecamente relacionado com a renormalização da constante de acoplamento gravitacional e conseqüentemente, para ser entendida precisa-se de um conhecimento melhor do comportamento ultravioleta da teoria.

\subsubsection{Buracos Negros em $D=4$ e $D=5$ com cargas。}

Nesta seção consideramos um buraco negro também em $D=4$ mas com quatro cargas [18]. Este tipo de métrica tem sido obtida no contexto de teorias de cordas [53]. Neste caso o elemento de linha é

$$
d s^{2}=-\lambda^{-1 / 2} h d t^{2}+\lambda^{1 / 2}\left[h^{-1} d r^{2}+r^{2}\left(d \theta^{2}+\sin ^{2} \theta d \varphi^{2}\right)\right],
$$

onde $h(r)$ tem a forma identica ao buraco de Schwarzschild, ou seja,

$$
h(r)=1-\frac{r_{0}}{r}
$$


enquanto a função $\lambda(r)$ que caracteriza as cargas do buraco negro é dada pela expressão

$$
\lambda(r)=\left(1+\frac{r_{0} \sinh ^{2} \alpha_{2}}{r}\right)\left(1+\frac{r_{0} \sinh ^{2} \alpha_{5}}{r}\right)\left(1+\frac{r_{0} \sinh ^{2} \alpha_{6}}{r}\right)\left(1+\frac{r_{0} \sinh ^{2} \alpha_{p}}{r}\right) .
$$

Obviamente o fator $\lambda(r)$ não modifica relevantemente o cálculo da energia livre perto do horizonte. Repetindo os cálculos anteriormente apresentados obtemos para a entropia

$$
S_{\epsilon}=\beta^{2} \frac{\partial F_{\epsilon}}{\partial \beta}=\frac{8 \pi^{3}}{45 \epsilon} \frac{\lambda^{3 / 2}\left(r_{0}\right) r_{0}^{4}}{\beta^{3}}
$$

Neste caso a aceleração gravitacional na superficie do buraco negro é

$$
\kappa_{s}=\frac{1}{2 r_{0} \lambda^{1 / 2}\left(r_{0}\right)}
$$

Portanto,

$$
\beta_{H}=4 \pi r_{0} \lambda^{1 / 2}\left(r_{0}\right)
$$

e conseqüentemente

$$
S=\frac{r_{0}}{360 \epsilon}
$$

Novamente usamos a "distância invariante"

$$
\int d s=\int_{r_{0}}^{r_{0}+\epsilon} d r \frac{\lambda^{1 / 4}(r)}{\sqrt{1-r_{0} / r}}=\sqrt{4 \epsilon r_{0} \lambda^{1 / 2}\left(r_{0}\right)}
$$

e finalmente a entropia fica

$$
S=\frac{r_{0}}{360 \epsilon}=\frac{A}{1440 \pi \epsilon r_{0} \lambda^{1 / 2}\left(r_{0}\right)}=\frac{A}{360 \pi\left(\int d s\right)^{2}},
$$

onde $A=4 \pi r_{0}^{2} \lambda^{1 / 2}\left(r_{0}\right)$ e a área do horizonte.

Aqui simplesmente foi conferido que para uma métrica tipo (2.39) com um fator extra $\lambda(r)$, o método da parede funciona na primeira aproximação.

Agora mostraremos a aplicação do método apresentado anteriormente para outro caso particular de um buraco negro em cinco dimensões com três cargas [18]. Obviamente o cálculo é muito parecido. Neste caso o elemento de linha é

$$
d s_{5}^{2}=-f^{-2 / 3} h d t^{2}+f^{1 / 3}\left[h^{-1} d r^{2}+r^{2} d \Omega_{3}^{2}\right],
$$


onde

$$
h(r)=1-\frac{r_{0}^{2}}{r^{2}}
$$

e as cargas são representadas na função $f(r)$ como

$$
f(r)=\left(1+\frac{r_{0}^{2} \sinh ^{2} \alpha}{r^{2}}\right)\left(1+\frac{r_{0}^{2} \sinh ^{2} \gamma}{r^{2}}\right)\left(1+\frac{r_{0}^{2} \sinh ^{2} \sigma}{r^{2}}\right)
$$

Agora o Ansatz é $\Phi(t, r, \theta, \psi, \zeta)=e^{-i E t} R(r) Y(\theta, \psi, \zeta)$ e obtemos a equação radial

$$
f^{2 / 3} h^{-1} E^{2} R(r)+\frac{1}{f^{1 / 3} r^{3}} \partial_{r}\left[r^{3} h \partial_{r} R(r)\right]-\frac{l(l+2)}{f^{1 / 3} r^{2}} R(r)-m^{2} R(r)=0,
$$

com o correspondente número de onda radial

$$
K=f^{1 / 2}\left(1-\frac{r_{0}^{2}}{r^{2}}\right)^{-1} \sqrt{E^{2}-\left(1-\frac{r_{0}^{2}}{r^{2}}\right)\left(\frac{l(l+2)}{f r^{2}}+\frac{1}{f^{2 / 3}} m^{2}\right)}
$$

Repetindo o mesmo procedimento que no caso anterior achamos para a energia livre a integral

$$
\begin{aligned}
F= & \frac{1}{\beta} \int d l(l+1)^{2} \int d n_{r} \ln \left(1-e^{-\beta E}\right) \\
= & -\frac{1}{\pi} \int d l(l+1)^{2} \int d E \frac{1}{\exp (\beta E)-1} \int_{r_{0}+\epsilon}^{L} d r f^{1 / 2}(r) \\
& \times\left(1-\frac{r_{0}^{2}}{r^{2}}\right)^{-1} \sqrt{E^{2}-\left(1-\frac{r_{0}^{2}}{r^{2}}\right)\left(\frac{l(l+2)}{f r^{2}}+\frac{m^{2}}{f^{2 / 3}}\right)}
\end{aligned}
$$

Também a integral em $l$ pode ser feita explicitamente, resultando em três termos, ou seja,

$$
\begin{aligned}
& \int d l(l+1)^{2} \sqrt{E^{2}-\frac{h l(l+2)}{f r^{2}}-\frac{h m^{2}}{f^{2 / 3}}}= \\
& \quad \frac{f^{3 / 2} r^{3}\left(E^{2}-\frac{h m^{2}}{f^{2 / 3}}+\frac{h}{f r^{2}}\right)^{2}}{8 h^{3 / 2}}\left[\frac{\pi}{2}-\arcsin \left(\sqrt{\frac{h / f r^{2}}{E^{2}-\frac{h m^{2}}{f^{2 / 3}}+\frac{h}{f r^{2}}}}\right)\right]+ \\
& +\frac{f r^{2}\left(E^{2}-\frac{h m^{2}}{f^{2 / 3}}\right)^{3 / 2}}{4 h}-\frac{f r^{2}\left(E^{2}-\frac{h m^{2}}{f^{2 / 3}}+\frac{h}{f r^{2}}\right) \sqrt{E^{2}-\frac{h m^{2}}{f^{2 / 3}}}}{8 h}
\end{aligned}
$$


Primeiramente calcularemos a contribuição para a energia livre, na vizinhança do horizonte, do primeiro termo da equação anterior, ou seja,

$$
F_{\epsilon}^{(5)}=-\frac{r_{0}^{4} f^{2}\left(r_{0}\right)}{32} \int_{0}^{\infty} d E \frac{E^{4}}{\exp (\beta E)-1} \int_{(1+\bar{\epsilon})^{2}}^{\bar{L}^{2}} d y(y-1)^{-5 / 2},
$$

onde $y=r^{2} / r_{0}^{2}$.

Usando a fórmula

$$
\int_{0}^{\infty} d E \frac{E^{4}}{\exp (\beta E)-1}=\frac{24 \zeta(5)}{\beta^{5}}
$$

e fazendo a integração, obtemos

$$
F_{\epsilon}^{(5)}=-\frac{\zeta(5)}{2^{5 / 2} \epsilon^{3 / 2}} \frac{f^{2}\left(r_{0}\right) r_{0}^{11 / 2}}{\beta^{5}} .
$$

Portanto a entropia correspondente a este termo é

$$
S_{\epsilon}^{(5)}=\beta^{2} \frac{\partial F_{\epsilon}^{(5)}}{\partial \beta}=\frac{5 \zeta(5)}{2^{5 / 2} \epsilon^{3 / 2}} \frac{f^{2}\left(r_{0}\right) r_{0}^{11 / 2}}{\beta^{4}} .
$$

Para este buraco negro a temperatura de Hawking é

$$
\beta_{H}=2 \pi r_{0} f^{1 / 2}\left(r_{0}\right) .
$$

Usando este resultado a entropia pode ser reescrita como

$$
S_{\epsilon}^{(5)}=\frac{5 \zeta(5)}{2^{5 / 2} 16 \pi^{4}} \frac{r_{0}^{3 / 2}}{\epsilon^{3 / 2}} .
$$

Novamente precisa-se da distância invariante, que é

$$
\int d s=\int_{r_{0}}^{r_{0}+\epsilon} d r \frac{f^{1 / 6}(r)}{\sqrt{1-r_{0}^{2} / r^{2}}}=\sqrt{8 f^{1 / 3}\left(r_{0}\right) r_{0} \epsilon}
$$

então a contribuição divergente do campo escalar para a entropia correspondente ao primeiro termo de (2.52) é

$$
S_{\epsilon}^{(5)}=\frac{A}{C_{N} 8^{3 / 2} \epsilon^{3 / 2} r_{0}^{3 / 2} f^{1 / 2}\left(r_{0}\right)}=\frac{A}{C_{N}\left(\int d s\right)^{3}}
$$


onde $A=2 \pi^{2} r_{0}^{3} f^{1 / 2}\left(r_{0}\right)$ e $C_{N}=\frac{8 \pi^{6}}{5 \zeta(5)}$.

Portanto, a expressão final para a entropia, levando-se em conta uma contribuição "nua", fica sendo

$$
S_{b a r e}+S_{\epsilon}^{(5)}=\left(\frac{1}{4 G_{N}}+\frac{1}{C_{N}\left(\int d s\right)^{3}}\right) A=\frac{A}{4 G_{R}} .
$$

Agora repetimos os mesmos cálculos para os outros dos termos de (2.52), cuja energia livre é

$$
F_{2+3}^{(5)}=-\frac{r_{0}^{3} f^{3 / 2}(1)}{16 \pi} \int d E \frac{E^{3}}{\exp (\beta)-1} \int_{(1+\bar{\epsilon})^{2}}^{\bar{L}^{2}} d y(y-1)^{-2} .
$$

Usando (2.22) e fazendo a integração, obtemos

$$
F_{2+3}^{(5)}=-\frac{\pi^{3}}{480 \epsilon} \frac{r_{0}^{4} f^{3 / 2}\left(r_{0}\right)}{\beta^{4}} .
$$

A entropia correspondente a esses dois termos é

$$
S_{2+3}^{(5)}=\frac{r_{0}}{960 \epsilon}=\frac{r_{0}^{2} f^{1 / 3}}{960 \epsilon r_{0} f^{1 / 3}}=\frac{A^{2 / 3}}{D_{N}\left(\int d s\right)^{2}}
$$

onde $D_{N}=\left(2 \pi^{2}\right)^{2 / 3} 120$.

Um fato interessante é que, sem fazer considerações adicionais temos contribuições de ordem menor a partir da integral em $l$, coisa que não acontece em quatro dimensões.

Resumindo, calculamos a expressão para a entropia de campos escalares propagando-se ao redor de buracos negros com cargas em quatro e cinco dimensões. Nós achamos o termo divergente dominante, que é proporcional à área do buraco negro em ambos os casos. Em 5 dimensões há, contudo, um termo subdominante proporcional à potência $2 / 3$ da área, que em 5 dimensões tem uma dimensão menor que a área (a área neste caso tem 3 dimensões espaciais) representando portanto um hiper comprimento. Sendo uma divergência subdominante, deveríamos também poder renormalizar com uma constante, o que de fato não acontece. Portanto este é um caso onde a lei das áreas pode ter um contra exemplo, ou o método da parede de tijolos falha. 


\subsubsection{Método da Parede de Tijolos na gravidade de ordem superior.}

No começo da seção anterior foi mostrado o método da parede de tijolos para uma solução de buraco negro na gravidade de Einstein-Hilbert, que é linear na curvatura $R$. Nesta seção mostraremos o que acontece quando o método da parede de tijolos é aplicado a teorias da gravidade de ordem superior na curvatura.

A teoria de gravidade de ordem superior conhecida como teoria da gravidade de Lovelock $[25,26,27,28,29,30]$ é excepcional no sentido de que as equações de movimento tem somente derivadas da métrica até segunda ordem apesar de haver na densidade langrangiana potências superiores da curvatura. Esta teoria também é covariante e livre de fantasmas, do mesmo modo que a Teoria Geral da Relatividade.

A ação de Lanczos-Lovelock é um polinômio de grau $[d / 2]$ na curvatura. Escrever explicitamente os termos da ação e as equações de movimento é uma tarefa muito complicada. Como exemplo deste fato temos que a parte quártica da ação de Lovelock em $D=10$ envolve 25 termos [32]. Felizmente uma formulação mais compacta foi proposta em termos das formas diferenciais [33, 34, 35].

Portanto, a ação de Lovelock pode ser escrita como [30]

$$
I_{G}=\kappa \int \sum_{m=0}^{[d / 2]} \alpha_{m} L^{(m)},
$$

onde $\alpha_{m}$ são constantes arbitrarias e $L^{(m)}$ é dado por

$$
L^{(m)}=\epsilon_{a_{1} \cdots a_{d}} R^{a_{1} a_{2}} \cdots R^{a_{2 m-1} a_{2 m}} e^{a_{2 m+1}} \cdots e^{a_{d}} .
$$

$R^{a b}$ é a curvatura de Riemann dada por

$$
R^{a b}=d \omega^{a b}+\omega_{c}^{a} w^{c b},
$$

onde $w_{a b}$ é a 1-forma representando a conexão de spin, e $e^{a}$ é o vielbein. $\mathrm{O}$ produto wedge é considerado nesta secção. As equações de movimento do campo podem ser obtidas através da variação com respeito a $e^{a}$ e $w^{a b}$,

$$
\sum_{p}^{\left[\frac{d-1}{2}\right]} \alpha_{p}(d-2 p) \mathcal{E}_{a}^{p}=0
$$




$$
\sum_{p}^{\left[\frac{d-1}{2}\right]} \alpha_{p} p(d-2 p) \mathcal{E}_{a b}^{p}=0
$$

onde foram definidos

$$
\begin{aligned}
\mathcal{E}_{a}^{p} & :=\epsilon_{a b_{1} \cdots b_{d-1}} R^{b_{1} b_{2}} \cdots R^{b_{2 p-1} b_{2 p}} e^{b_{2 p+1}} \cdots e^{b_{d-1}} \\
\mathcal{E}_{a b}^{p} & :=\epsilon_{a b a_{3} \cdots a_{d}} R^{a_{3} a_{4}} \cdots R^{a_{2 p-1} a_{2 p}} T^{a_{2 p+1}} e^{a_{2 p+2}} \cdots e^{a_{d}} .
\end{aligned}
$$

e onde $T^{a}=d e^{a}+\omega_{b}^{a} e^{b}$ é a torsão.

Para uma dimensão dada e uma escolha arbitrária dos coeficientes $\alpha_{p}$ as teorias de Lovelock apresentam problemas. Um exemplo disto é que aparecem soluções com energia negativa que tem horizonte e também soluções com energia positiva que tem singularidades nuas [32]. No trabalho [30] foi apresentada uma escolha particular dos coeficientes $\alpha_{p}$ que levam a configurações de buracos negros bem definidos. Os coeficientes propostos foram

$$
\alpha_{p}:=c_{p}^{k}= \begin{cases}\frac{l^{2(p-k)}}{(d-2 p)}\left(\begin{array}{l}
k \\
p
\end{array}\right) & , p \leq k \\
0 & , p>k\end{cases}
$$

onde $1 \leq k \leq[(d-1) / 2]$.

Para uma dimensão $d$ dada, os coeficientes $c_{m}^{k}$ levam a uma família de teorias inequivalentes, rotuladas por $k$ que representa a potência maior da curvatura na Lagrangeana. Este conjunto de teorias só possue duas constantes fundamentais, $\kappa \mathrm{e} l$, relacionadas respectivamente à constante gravitacional $G_{k}$ e à constante cosmológica $\Lambda$,

$$
\begin{aligned}
& \kappa=\frac{1}{2(d-2) ! \Omega_{d-2} G_{k}}, \\
& \Lambda=-\frac{(d-1)(d-2)}{2 l^{2}} .
\end{aligned}
$$

Nós estamos interessados nas soluções de buracos negros que são assintóticamente chatas, portanto consideramos o limite onde a constante cosmológica é nula. Quando $l \rightarrow \infty$ o único termo que não se anula na $\mathrm{Eq}(2.65)$ é o k-ésimo; então a ação é obtida a partir da $\mathrm{Eq}(2.65)$ com os coefficientes

$$
\alpha_{p}:=\tilde{c}_{p}^{k}=\frac{1}{(d-2 k)} \delta_{p}^{k} .
$$


Neste caso a ação fica

$$
\tilde{I}_{k}=\frac{\kappa}{(d-2 k)} \int \epsilon_{a_{1} \cdots a_{d}} R^{a_{1} a_{2}} \cdots R^{a_{2 k-1} a_{2 k}} e^{a_{2 k+1}} \cdots e^{a_{d}} .
$$

Note-se que para $k=1$ a ação de Einstein sem constante cosmológica é obtida. Entretanto, para $k=2$ temos a ação de Gauss-Bonnet,

$$
I_{2}=\frac{(d-2) ! \kappa}{(d-4)} \int d^{d} x \sqrt{-g}\left(-R_{\mu \nu \alpha \beta} R^{\mu \nu \alpha \beta}+4 R_{\mu \nu} R^{\mu \nu}-R^{2}\right)
$$

A existência de soluções de buraco negro é usada como teste para conferir a validade destas teorias. Varios autores tem encontrado soluções esfericamente simétricas na gravidade de Lovelock mas sem escolha para os coeficientes $[32,36,37]$. Agora mostramos uma solução para esta teoria, com $\Lambda \neq 0$.

Em um sistema de coordenadas apropriado a métrica pode ser escrita como

$$
d s^{2}=-N^{2}(r) f^{2}(r) d t^{2}+\frac{d r^{2}}{f^{2}(r)}+r^{2} d \Omega_{d-2}^{2}
$$

Trabalhar com Hamiltonianas é uma forma simples de se calcular a métrica (2.78). Aplicando os métodos de M. Bañados et al [31, 30] a ação (2.65) toma a forma (ver Apêndice B)

$$
I=\Delta t \int \frac{N}{2} \frac{d}{d r}\left\{\frac{r^{d-1}}{G_{k}}\left[F(r)+\frac{1}{\ell^{2}}\right]^{k}\right\} d r+B_{E}
$$

onde

$$
F(r)=\frac{1-f^{2}(r)}{r^{2}}
$$

e $B_{E}$ é o termo de fronteira. Então as equações de movimento são

$$
\begin{aligned}
\frac{d N}{d r} & =0 \\
\frac{d}{d r}\left(r^{d-1}\left[F(r)+\frac{1}{l^{2}}\right]^{k}\right) & =0
\end{aligned}
$$

tendo como soluções

$$
\begin{aligned}
& N=N_{\infty} \\
& f^{2}=1+\frac{r^{2}}{\ell^{2}}-\left(\frac{2 G_{k} M}{r^{d-2 k-1}}\right)^{1 / k} .
\end{aligned}
$$


Desta forma, podemos escrever a métrica como

$$
\begin{aligned}
d s^{2}= & -\left(1+\frac{r^{2}}{\ell^{2}}-\left(\frac{2 G_{k} M}{r^{d-2 k-1}}\right)^{1 / k}\right) d t^{2}+ \\
& \frac{d r^{2}}{1+\frac{r^{2}}{\ell^{2}}-\left(\frac{2 G_{k} M}{r^{d-2 k-1}}\right)^{1 / k}}+r^{2} d \Omega_{d-2}^{2}
\end{aligned}
$$

No limite quando a constante cosmológica é zero $(\ell \rightarrow \infty)$ a solução de buraco negro é ${ }^{1}$

$$
d s^{2}=-\left(1-\left(\frac{r_{h}}{r}\right)^{\gamma}\right) d t^{2}+\frac{d r^{2}}{1-\left(\frac{r_{h}}{r}\right)^{\gamma}}+r^{2} d \Omega_{d-2}^{2},
$$

onde $r_{h}=\left(2 G_{k} M\right)^{1 /(d-2 k-1)}$ é o raio do horizonte de eventos e

$$
\gamma=\frac{d-2 k-1}{k}
$$

Para a métrica (2.86) a temperatura de Hawking é

$$
T=\frac{\gamma}{4 \pi r_{h}} .
$$

Para calcular a entropia usamos o fato conhecido que a função de partição que descreve a termodinâmica do buraco negro é obtida, através da integral de caminho Euclidiana na aproximação de ponto de sela perto da solução de buraco negro, ou seja,

$$
Z \approx e^{-I_{E}}
$$

Isto significa que a ação Euclidiana calculada na configuração do buraco negro é identificada com a energia livre multiplicada por $\beta$,

$$
I_{E}=\beta F=\beta M-S .
$$

Fazendo uma rotação de Wick na ação (2.79), e considerando $\beta=\Delta \tau$ obtemos

$$
I_{E}=-\beta \int_{r_{h}}^{\infty} \frac{N}{2} \frac{d}{d r}\left\{\frac{r^{d-1}}{G_{k}} F^{k}(r)\right\} d r+B_{E} .
$$

A parte volumétrica da ação é uma combinação dos vínculos, portanto seu valor "on-shell" é dado pelo termo de fronteira $B_{E}$. Este termo pode

${ }^{1} \mathrm{O}$ caso $\mathrm{d}-2 \mathrm{k}-1=0$ é excluido. 
ser determinado levando-se em conta que $I_{E}$ é estacionária na geometria do buraco negro. Variando (2.91) chegamos a

$$
0=\delta I_{E}=-\frac{\beta N}{2 G_{k}} \int_{r_{h}}^{\infty} \frac{d}{d r}\left\{r^{d-1} \delta\left([F(r)]^{k}\right)\right\} d r+\delta B_{E} \quad,
$$

calculada "on shell". A partir desta expressão encontramos

$$
0=-\left.\frac{\beta N}{2 G_{k}}\left\{r^{d-1} \delta\left([F(r)]^{k}\right)\right\}\right|_{r_{h}} ^{\infty}+\delta B_{E}(\infty)+\delta B_{E}\left(r_{h}\right) .
$$

Consideramos inicialmente o termo correspondente à região $(r \rightarrow \infty)$. Levandose em conta $(2.80)$ e $(2.84)$ obtemos

$$
\delta B_{E}(\infty)=\beta \delta M .
$$

Agora calculamos o termo correspondente ao horizonte

$$
\delta B_{E}\left(r_{h}\right)=-\frac{\beta N}{2 G_{k}} r_{h}^{d-1} \delta\left(\left[F\left(r_{h}\right)\right]^{k}\right)
$$

Mas

$$
\delta\left(\left[F\left(r_{h}\right)\right]^{k}\right)=\left(\frac{\partial\left(\left[F\left(r_{h}\right)\right]^{k}\right)}{\partial f^{2}}\right)_{r=r_{h}}\left(\frac{d f^{2}}{d r}\right)_{r=r_{h}} .
$$

Calculando esta expressão e lembrando que $\left.\frac{d f^{2}}{d r}\right|_{r_{h}}=\frac{4 \pi}{\beta}$ obtemos

$$
\delta B_{E}\left(r_{h}\right)=-\frac{2 \pi k}{G_{k}} r_{h}^{d-2 k-1} \delta r_{h}
$$

Recordando mais uma vez que $\beta F=B_{E}(\infty)+B_{E}\left(r_{h}\right)$ finalmente chegamos à expressão para a entropia

$$
S_{k}=\frac{2 \pi k}{G_{k}} \int_{0}^{r_{h}} r^{d-2 k-1} d r=\frac{2 \pi k}{G_{k}} \frac{r_{h}^{d-2 k}}{d-2 k} .
$$

Isto é, uma função crescente de $r_{h}$, o que é consistente com a segunda lei da termodinâmica. Esta expressão da entropia não satifaz à lei da área. Portanto o comportamento "holográfico" parece ser uma propriedade única da Relatividade Geral. 
Agora, gostaríamos de aplicar o método do 't Hooft para este tipo de buracos negros. Como exemplo realizaremos os cálculos para um buraco negro em oito dimensões e $k=2$ com elemento de linha

$$
d s^{2}=-h d t^{2}+h^{-1} d r^{2}+r^{2} d \Omega_{4}^{2},
$$

onde

$$
h=1-\left(\frac{r_{h}}{r}\right)^{3 / 2} .
$$

Neste caso, o Ansatz para a equação de Klein-Gordon em oito dimensões é

$$
\Phi(t, r, \theta)=e^{-i E t} R(r) Y(\theta),
$$

onde $\theta$ representa todas as variáveis angulares. A equação angular é

$$
\triangle Y(\theta)=-l(l+5) Y(\theta) .
$$

Então a equação radial fica

$$
h^{-1} E^{2} R(r)+\frac{1}{r^{6}} \partial_{r}\left[r^{6} h \partial_{r} R(r)\right]-\frac{l(l+5)}{r^{2}} R(r)-m^{2} R(r)=0
$$

e conseqüentemente o número radial é

$$
K=\left(1-\left(\frac{r_{h}}{r}\right)^{3 / 2}\right)^{-1} \sqrt{E^{2}-\left(1-\left(\frac{r_{h}}{r}\right)^{3 / 2}\right)\left(\frac{l(l+5)}{r^{2}}+m^{2}\right)} .
$$

Depois de repetir os mesmos passos que nos casos anteriores, obtemos para a energia livre

$$
\begin{aligned}
F & =\frac{1}{\beta} \sum \ln \left(1-e^{-\beta E}\right) \\
& =\frac{1}{\beta} \int d l D_{l} \int d n_{r} \ln \left(1-e^{-\beta E}\right)
\end{aligned}
$$

onde

$$
D_{l}=\frac{(2 l+5)(l+4) !}{5 ! l !}=\frac{(2 l+5)(l+1)(l+2)(l+3)(l+4)}{5 !}
$$

é a degenerescência dos modos esféricos [39]. 
Integrando por partes e usando (2.11) obtemos

$$
\begin{aligned}
F= & -\int d l D_{l} \int d E \frac{1}{\exp (\beta E)-1} n_{r} \\
= & -\frac{1}{\pi} \int d l D_{l} \int d E \frac{1}{\exp (\beta E)-1} \int_{r_{h}+\epsilon}^{L} d r \\
& \times\left(1-\left(\frac{r_{h}}{r}\right)^{3 / 2}\right)^{-1} \sqrt{E^{2}-\left(1-\left(\frac{r_{h}}{r}\right)^{3 / 2}\right)\left(\frac{l(l+5)}{r^{2}}+m^{2}\right)}
\end{aligned}
$$

Novamente, a integração na variável $l$ pode ser feita explicitamente,

$$
\begin{aligned}
& \int d l D_{l} \sqrt{E^{2}-\left(1-\left(\frac{r_{h}}{r}\right)^{3 / 2}\right)\left(\frac{l(l+5)}{r^{2}}+m^{2}\right)}= \\
& \quad \frac{16 r^{6}\left(E^{2}-h m^{2}\right)^{7 / 2}}{5 ! 105 h^{3}}+\frac{8 r^{4}\left(E^{2}-h m^{2}\right)^{5 / 2}}{5 ! 3 h^{2}}+\frac{16 r^{2}\left(E^{2}-h m^{2}\right)^{3 / 2}}{5 ! h}-(2.1
\end{aligned}
$$

Nós estamos interessados na contribuição principal para a energia livre, perto do horizonte. Portanto ficamos só com o primeiro termo da equação anterior, que é

$$
F=-\frac{16}{5 ! 105 \pi} \int d E \frac{1}{\exp (\beta E)-1} \int_{r_{h}+\epsilon}^{L} d r r^{6} h^{-4}\left[E^{2}-h m^{2}\right]^{7 / 2}
$$

Introduzindo a variável $y=\left(\frac{r}{r_{h}}\right)^{3 / 2}$ e substituindo em (2.109) temos

$F=-\frac{32 r_{h}^{7}}{5 ! 315 \pi} \int d E \frac{1}{\exp (\beta E)-1} \int_{(1+\bar{\epsilon})^{3 / 2}}^{\bar{L}^{3 / 2}} d y y^{11 / 3}\left(1-\frac{1}{y}\right)^{-4}\left[E^{2}-\left(1-\frac{1}{y}\right) m^{2}\right]^{7 / 2}$

onde $\bar{\epsilon}=\frac{\epsilon}{r_{h}}, \bar{L}=\frac{L}{r_{h}}$.

Perto do horizonte, para $y \rightarrow 1$, achamos [16]

$$
F=-\frac{32 r_{h}^{7}}{5 ! 315 \pi} \int_{0}^{\infty} d E \frac{E^{7}}{\exp (\beta E)-1} \int_{(1+\bar{\epsilon})^{3 / 2}}^{\bar{L}^{3 / 2}} d y(y-1)^{-4}
$$

Usando a fórmula

$$
\int_{0}^{\infty} d E \frac{E^{7}}{\exp (\beta E)-1}=\frac{7 ! \zeta(8)}{\beta^{8}}
$$


e integrando em $y$ a expressão para $F$ fica

$$
F=-\frac{2^{9} \zeta(8)}{45 \pi 3^{3}} \frac{r_{h}^{10}}{\epsilon^{3} \beta^{8}}
$$

permitindo-nos calcular a entropia,

$$
S=\beta^{2} \frac{\partial F}{\partial \beta}=\frac{2^{12} \zeta(8)}{45 \pi 3^{3}} \frac{r_{h}^{10}}{\epsilon^{3} \beta^{7}} .
$$

O inverso da temperatuta de Hawking é

$$
\beta=\frac{8 \pi}{3} r_{h}
$$

e, finalmente, a entropia é

$$
S=\frac{3^{4} \zeta(8)}{45 \pi^{8} 2^{9}} \frac{r_{h}^{3}}{\epsilon^{3}}
$$

Usando a distância invariante

$$
\int d s=\int_{r_{h}}^{r_{h}+\epsilon} d r \frac{1}{\sqrt{1-\left(r_{h} / r\right)^{3 / 2}}}=\sqrt{\frac{8 r_{h} \epsilon}{3}},
$$

a entropia pode ser reescrita como

$$
S=\frac{\zeta(8)}{15 \pi^{8}} \frac{r_{h}^{6}}{\left(r_{h} \epsilon\right)^{3}}=\frac{A}{D_{(8)}^{(2)}\left(\int d s\right)^{6}},
$$

onde $A=\frac{16}{15} \pi^{3} r_{h}^{6}$ é a área do horizonte e $D_{(8)}^{(2)}=\frac{2^{4} \pi^{11}}{\zeta(8)}$.

Portanto a entropia do campo escalar é proporcional a área e diverge cubicamente com o corte $\epsilon$.

Já foi demonstrado que a questão da finitude da entropia pode ser resolvida pela renormalização da constante de Newton. Aqui, isto não é possível tendo em vista que a entropia nua (2.98) não depende do mesmo modo do raio do horizonte, em comparação com o termo divergente (2.118).

Repetindo o mesmo procedimento podemos encontrar a expressão geral da energia livre para $d$ e $k$ dados,

$$
F_{\epsilon}^{(d)}=-C_{(d)}^{(k)} \frac{r_{h}^{\kappa_{d}}}{\epsilon^{\frac{d-2}{2}} \beta^{d}}
$$


onde $\kappa_{d}=d+\frac{d-4}{2}$ e $C_{(d)}^{(k)}$ são as constantes calculadas para valores de $d$ e $k$ dados (ver Apêndice C). Lembrando que $\beta=\frac{4 \pi r_{h}}{\gamma}$ e levando em conta que a distância invariante no caso geral é

$$
\int d s=\int_{r_{h}}^{r_{h}+\epsilon} d r \frac{1}{\sqrt{1-\left(r_{h} / r\right)^{\gamma}}}=\sqrt{\frac{4 r_{h} \epsilon}{\gamma}}
$$

a entropia fica

$$
S^{(d)}=\frac{d C_{(d)}^{(k)} \gamma^{d / 2} 2^{d-2}}{(4 \pi)^{d-1}} \frac{r^{d-2}}{\left(\int d s\right)^{d-2}}=\frac{A}{D_{(d)}^{(k)}\left(\int d s\right)^{d-2}}
$$

onde $A=\frac{2 \pi^{(d-1) / 2}}{\Gamma((d-1) / 2)} r^{d-2}$ e $D_{(d)}^{(k)}=\frac{2^{d+1} \pi^{3 / 2(d-1)}}{d C_{(d)}^{(k)} \gamma^{d / 2} \Gamma((d-1) / 2)}$.

Este resultado mostra que o método da parede de tijolo só se adequa ao caso linear. A entropia (2.121) não coincide com a expressão (2.98).

\subsection{Entropia e Teoria Conforme.}

Nos últimos anos tem havido vários trabalhos indicando que a simetria conforme bidimensional pode ser revelante na descrição dos estados do buraco negro $[9,45]$. As simetrias determinam muitas propriedades de uma teoria quântica, mas ordinariamente não se pensa que a densidade de estados seja uma dessas propriedades. Entretanto no caso das teorias de campo conforme bidimensionais o grupo de simetria faz precisamente isto, determina a densidade de estados. Tendo a algebra de Virasoro é possível ter a densidade de estados com a conhecida fórmula de Cardy [44]. Usando estas ferramentas tem sido apresentadas duas estratégias para investigar a mecânica estatística da entropia de um buraco negro. Primeiramente podemos tentar reduzir dimensionalmente a teoria geral da relatividade a duas dimensões na vizinhança do horizonte de eventos e olhar se é possivel identificar uma teoria de campos conforme e determinar sua correspondente carga central. Outra alternativa é ver a álgebra de Poisson dos geradores dos difeomorfismos e procurar se um subgrupo apropriado de transformações no plano $r-t$ adquire carga central.

Nesta seção mostramos a via adotada por Solodukhin, usando a primeira estratégia. Ele mostrou que a redução dimensional da (GR) perto do horizonte leva a uma teoria de Liouville com uma carga central calculavel. Primeiramente fazemos um resumo das características fundamentais das teorias conformes. 


\subsubsection{Algebra de Virasoro e a Fórmula de Cardy.}

Nesta seção mostraremos algumas propriedades das teorias conformes, que são um tipo particular de teoria quântica de campos. A propriedade característica deste tipo de teoria é que são invariantes perante as transformações conformes. Este é um tipo de transformação de coordenadas, $x \rightarrow \tilde{x}$ que deixa a métrica invariante a menos de um fator de escala,

$$
g_{\mu \nu}(x) \rightarrow g_{\mu \nu}(\tilde{x})=e^{\omega(x)} g_{\mu \nu}(x) .
$$

Estas transformações formam um grupo que é conhecido como grupo conforme. Também é conhecido que estas transformações preservam o ângulo $A \cdot B / \sqrt{A^{2} B^{2}}$ entre dois vetores quaisquer $A_{\mu}$ e $B_{\mu}$.

O grupo conforme é composto pelas seguintes transformações:

1) Translações

$$
x^{\mu} \rightarrow \tilde{x}^{\mu}=x^{\mu}+a^{\mu}
$$

2) Rotações

$$
x^{\mu} \rightarrow \tilde{x}^{\mu}=\Lambda_{\nu}^{\mu} x^{\nu} \quad \Lambda_{\nu}^{\mu} \in S O(d)
$$

3) Transformações de escala

$$
x^{\mu} \rightarrow \tilde{x}^{\mu}=\lambda x^{\mu}
$$

4) Transformações Conformes Especiais

$$
x^{\mu} \rightarrow \tilde{x}^{\mu}=\frac{x^{\mu}+b^{\mu} x^{2}}{1+2 b \cdot x+b^{2} x^{2}} .
$$

Agora, para obter os geradores das transformações infinitesimais do grupo conforme consideramos a transformação infinitesimal

$$
x^{\mu} \rightarrow x^{\mu}+\epsilon^{\mu}\left(x^{\mu}\right) .
$$

Levando-se em conta a propriedade de transformação de um tensor simétrico de posto dois,

$$
\tilde{g}_{\mu \nu}(\tilde{x})=\frac{\partial x^{\lambda}}{\partial \tilde{x}^{\mu}} \frac{\partial x^{\rho}}{\partial \tilde{x}^{\nu}} g_{\lambda \rho},
$$

e considerando a métrica de referência $g_{\mu \nu}=\delta_{\mu \nu}$, assim como a equação (2.122) obtemos

$$
\partial_{\mu} \epsilon_{\nu}+\partial_{\nu} \epsilon_{\mu}=\frac{2}{d}(\partial \cdot \epsilon) \delta_{\mu \nu}
$$


No caso de duas dimensões a equação (2.129) equivale às condições de Cauchy-Riemann

$$
\partial_{1} \epsilon_{1}=\partial_{2} \epsilon_{2}, \quad \partial_{1} \epsilon_{2}=-\partial_{2} \epsilon_{1} .
$$

Estas condições, em função das coordenadas complexas

$$
z=x_{1}+i x_{2}, \quad \bar{z}=x_{1}-i x_{2} \quad,
$$

significam que temos as funções analíticas (dependência holomórfica)

$$
\epsilon(z)=\epsilon_{1}+i \epsilon_{2} \quad e \quad \bar{\epsilon}(\bar{z})=\epsilon_{1}-i \epsilon_{2} \quad .
$$

Portanto as transformações conformes em duas dimensões podem ser identificadas com as transformações de coordenadas analíticas

$$
z \rightarrow f(z) \quad \bar{z}=\bar{f}(\bar{z})
$$

Uma transformação conforme (holomórfica) infinitesimal pode ser expressa como

$$
z^{\prime}=z+\epsilon(z), \quad \epsilon(z)=\sum c_{n} z^{n+1},
$$

onde $\epsilon$ admite uma expansão de Laurent ao redor de $z=0$. O efeito desta transformação num campo sem spin e dimensão é

$$
\delta \phi=\sum c_{n} \ell_{n} \phi(z, \bar{z})+\bar{c}_{n} \bar{\ell}_{n} \phi(z, \bar{z}),
$$

onde introduzimos os geradores

$$
\ell_{n}=-z^{n+1} \partial_{z}, \quad \bar{\ell}=-\bar{z}^{n+1} \partial_{\bar{z}} .
$$

Estes geradores obedecem as seguintes relações de comutação

$$
\begin{aligned}
{\left[\ell_{n}, \ell_{m}\right] } & =(n-m) \ell_{n+m}, \\
{\left[\bar{\ell}_{n}, \bar{\ell}_{m}\right] } & =(n-m) \bar{\ell}_{n+m}, \\
{\left[\ell_{n}, \bar{\ell}_{m}\right] } & =0 .
\end{aligned}
$$

Esta é conhecida como a Álgebra de Witt.

Mas esta álgebra recebe uma correção quando se faz a quantização, devido à ambiguidade no ordenamento normal,

$$
\left[L_{n}, L_{m}\right]=(n-m) L_{n+m}+A(n) \delta_{m+n, 0} .
$$


Esta álgebra generalizada é conhecida como a extensão central da álgebra de Witt. Os coeficientes $A(n)$ podem ser determinados a partir da identidade de Jacobi

$$
\left[L_{k},\left[L_{n}, L_{m}\right]\right]+\left[L_{n},\left[L_{m}, L_{k}\right]\right]+\left[L_{m},\left[L_{k}, L_{n}\right]\right]=0
$$

para os commutadores. Depois de um pouco de trabalho se obtém

$$
A_{n}=\frac{c}{12} n\left(n^{2}-1\right),
$$

então temos

$$
\left[L_{m}, L_{n}\right]=(m-n) L_{m+n}+\frac{c}{12} m\left(m^{2}-1\right) \delta_{m+n, 0}
$$

que é conhecida como a álgebra de Virasoro [43], e $c$ é conhecido como carga central ou anomalia conforme.

Também é conveniente saber a dimensão de uma representação de uma álgebra de Virasoro dada. Isto pode ser comparado com o caso mais conhecido que é o grupo $S U(2)$. Tendo o peso maior $m$, sabemos que a dimensão da representação é $2 m+1$. No caso da álgebra de Virasoro uma fórmula equivalente foi dada por Cardy [44]. Se temos uma representação da álgebra de Virasoro com carga central $c$ e peso máximo $l$, ou seja

$$
L_{0}|l>=l| l>
$$

e $L_{n} \mid l>=0$, para $n>0$, a dimensão da representação (número de estados) é

$$
d=\exp \left(2 \pi \sqrt{\frac{c l}{6}}\right)
$$

com uma entropia correspondente

$$
S=2 \pi \sqrt{\frac{c l}{6}} .
$$

Esta fórmula é conhecida na literatura como a fórmula de Cardy.

Para obter este resultado [46] se considera a função de partição num torus

$$
Z(\tau, \bar{\tau})=\operatorname{Tr} e^{i 2 \pi \tau L_{0}} e^{-i 2 \pi \bar{\tau} \bar{L}_{0}}=\sum \rho e^{i 2 \pi \tau \Delta} e^{-i 2 \pi \bar{\tau} \bar{\Delta}} .
$$


Para uma teoria unitaria, $\rho$ é o número de estados com autovalores $L_{0}=\Delta$, $\bar{L}_{0}=\bar{\Delta}$.

Então, conhecendo a função de partição podemos determinar a densidade de estados por integração. Considerando $\tau$ e $\bar{\tau}$ como variáveis independentes e colocando $q=e^{2 \pi i \tau}$ e $\bar{q}=e^{2 \pi i \bar{\tau}}$ temos

$$
\rho=\frac{1}{(2 \pi i)^{2}} \int \frac{d q}{q^{\Delta+1}} \frac{d \bar{q}}{\bar{q}^{\bar{\Delta}+1}} Z(q, \bar{q})
$$

onde as integrais são nos contornos que fecham $q=0$ e $\bar{q}=0$. Obviamente poucas vezes conhecemos $Z(q, \bar{q})$, mas Cardy mostrou que a grandeza

$$
Z_{0}=\operatorname{Tr} e^{2 \pi i\left(L_{0}-\frac{c}{24}\right) \tau} e^{-2 \pi i\left(\bar{L}_{0}-\frac{c}{24}\right) \bar{\tau}}
$$

é invariante sob a transformação $\tau \rightarrow-1 / \tau$. Portanto levando-se em conta este fato e considerando só a dependência em $\tau$ temos

$$
Z(\tau)=e^{\frac{2 \pi i c}{24} \tau} Z_{0}(-1 / \tau)=e^{\frac{2 \pi i c}{24} \tau} e^{\frac{2 \pi i c}{24} \tau} \frac{1}{\tau}(-1 / \tau),
$$

e

$$
\rho(\Delta)=\int d \tau e^{-2 \pi i \Delta \tau} e^{\frac{2 \pi i c}{24} \tau} e^{\frac{2 \pi i c}{24} \frac{1}{\tau}} Z(-1 / \tau)
$$

A chave na aproximação do ponto de sela é separar o integrando numa fase que varía rapidamente e um prefator quasi constante. Assumiremos que $Z(-1 / \tau)$ varia pouco perto do valor extremo da fase. Para um valor grande de $\Delta$, o extremo do expoente é

$$
\tau=i \sqrt{c / 24 \Delta}
$$

Substituindo em (2.151) obtemos

$$
\rho(\Delta) \approx \exp \left(2 \pi \sqrt{\frac{c \Delta}{6}}\right) Z(i \infty)
$$

Conferindo a aproximação de ponto de sela temos que no ponto $\tau=\frac{i}{\epsilon}$

$$
Z(i / \epsilon)=\sum \rho(\Delta) e^{-2 \pi \Delta / \epsilon}
$$

se o menor autovalor de $L_{0}$ for $\Delta_{0}=0$, então $Z(i / \epsilon)$ fica sendo uma constante para $\epsilon \rightarrow 0$ e portanto a aproximação de ponto de sela é boa. 


\subsubsection{Teoria conforme na Relatividade Geral.}

Consideremos uma métrica quadridimensional e esféricamente simétrica com forma geral [45],

$$
d s^{2}=\gamma_{a b} d x^{a} d x^{b}+r^{2}\left(d \theta^{2}+\sin ^{2} \theta d \phi^{2}\right),
$$

onde $\gamma_{a b}$ pode ser considerada a métrica no espaço bidimensional $M^{2}(t, x)$. Esta tem a forma

$$
d s_{(2)}^{2}=\gamma_{a b} d x^{a} d x^{b}=-g(x) d t^{2}+\frac{d x^{2}}{g(x)}
$$

com a função $g(x)$ anulando-se em $x=x_{h}$ onde está localizado o horizonte. Uma forma simples que pode ter esta função é

$$
g(x)=\frac{2}{\beta_{H}}\left(\left(x-x_{h}\right)+O\left(x-x_{h}^{2}\right)^{2}\right),
$$

onde $\beta_{H}$ é uma constante relacionada com a aceleração de gravidade na superficie.

Então consideremos a ação de Einstein-Hilbert

$$
W_{E H}=-\frac{1}{16 \pi G} \int_{M^{4}} d^{4} z \sqrt{-g} R_{(4)}
$$

calculada na métrica (2.155). Integrando nas variaveis angulares chegamos a uma teoria efetiva em duas dimensões descrita pela ação

$$
W=-\int_{M^{2}} d^{2} x \sqrt{-\gamma}\left(\frac{1}{2}(\nabla \Phi)^{2}+\frac{1}{4} \Phi^{2} R+\frac{1}{2 G}\right),
$$

onde $\Phi=r G^{-1 / 2}$ e $R$ é a curvatura escalar em duas dimensões. Esta ação pode ser transformada e levada a uma forma similar à ação de Liouville mediante a transformação

$$
\gamma_{a b}=\left(\frac{\phi_{h}}{\phi}\right)^{\frac{1}{2}} e^{\frac{2}{q_{\Phi_{h}}} \phi} \bar{\gamma}_{a b}, \quad \phi=\frac{1}{q} \frac{\Phi^{2}}{\Phi_{h}},
$$

onde $\Phi_{h}=r_{h} G^{-1 / 2}$ é o valor clássico do campo no horizonte. Portanto, a ação fica

$$
W=-\int_{M^{2}} d^{2} x\left(\frac{1}{2}(\nabla \phi)^{2}+\frac{1}{4} q \Phi_{h} \phi R+U(\phi)\right),
$$


com potencial

$$
U(\phi)=\frac{1}{2 G}\left(\frac{\phi_{h}}{\phi}\right)^{\frac{1}{2}} e^{\frac{2}{q \Phi_{h}} \phi} .
$$

Variando a ação (2.161) com respeito a $\phi$ obtemos a equação

$$
\square \phi=\frac{1}{4} q \Phi_{h} R+U^{\prime}(\phi)
$$

enquanto a variação em relação à métrica $\gamma_{a b}$ leva aos vínculos

$$
T_{a b} \equiv \frac{1}{2} \partial_{a} \phi \partial_{b} \phi-\frac{1}{4} \bar{\gamma}_{a b}(\nabla \phi)^{2}+\frac{1}{4} q \Phi_{h}\left(\bar{\gamma}_{a b} \square \phi-\nabla_{a} \nabla_{b} \phi\right)-\frac{1}{2} \bar{\gamma}_{a b} U(\phi)=0
$$

A teoria do campo escalar $\phi$ descrita pela ação (2.161) não é conformalmente invariante. Note-se que o traço de (2.164),

$$
\bar{\gamma}^{a b} T_{a b}=\frac{1}{4} q \Phi_{h} \square \phi-U(\phi)
$$

não se anula. Entretanto a teoria se torna invariante conforme quando é considerada numa distância infinitesimal perto do horizonte. Neste caso a métrica bidimensional toma a forma (2.156). Com esta métrica é conveniente usar a coordenada

$$
z=\int^{x} \frac{d x}{g(x)}=\frac{\beta_{H}}{2} \ln \left(x-x_{h}\right)
$$

de modo que na vizinhança do horizonte $\left(x-x_{h}\right.$ pequeno) $z$ tende a menos infinito. A função $g$ da métrica toma a forma

$$
g(z)=g_{0} e^{\frac{2}{\beta_{H}} z}
$$

e se anula exponencialmente no horizonte. Nas coordenadas $(t, z)$ a equação (2.163) fica

$$
-\partial_{t}^{2} \phi+\partial_{z}^{2} \phi=\frac{1}{4} q \Phi_{h} R g(z)+g(z) U^{\prime}(\phi)
$$

$\mathrm{Na}$ região muito perto do horizonte $(z \rightarrow-\infty)$ o lado direito de (2.168) é desprezível e obtemos a equação

$$
\partial_{t}^{2} \phi-\partial_{z}^{2} \phi=0
$$


que descreve o campo livre propagando-se no espaço-tempo chato com coordenadas $(t, z)$. Expresando os vínculos (2.164) em função das coordenadas $(t, z)$ encontramos

$$
\begin{aligned}
& T_{00}=\frac{1}{4}\left(\left(\partial_{t} \phi\right)^{2}+\left(\partial_{z} \phi\right)^{2}\right)-\frac{q \Phi_{h}}{4}\left(\partial_{z}^{2} \phi-\frac{g_{x}^{\prime}}{2} \partial_{z} \phi\right)+\frac{1}{2} g(z) U(\phi) \\
& T_{0 z}=\frac{1}{2} \partial_{t} \phi \partial_{z} \phi-\frac{q \Phi_{h}}{4}\left(\partial_{z} \partial_{t} \phi-\frac{g_{x}^{\prime}}{2} \partial_{t} \phi\right) \\
& T_{z z}=\frac{1}{4}\left(\left(\partial_{t} \phi\right)^{2}+\left(\partial_{z} \phi\right)^{2}\right)+\frac{q \Phi_{h}}{4}\left(-\partial_{t}^{2} \phi+\frac{g_{x}^{\prime}}{2} \partial_{z} \phi\right)-\frac{1}{2} g(z) U(\phi)
\end{aligned}
$$

O traço é

$$
-T_{00}+T_{z z}=\frac{1}{4} q \Phi_{h}\left(-\partial_{t}^{2} \phi+\partial_{z}^{2} \phi\right)-g(z) U(\phi) .
$$

Quando $z$ tende a menos infinito este traço se anula, usando a equação de movimento (2.169). Isto, em particular, garante que a álgebra de Poisson dos vínculos fecha (2.171) e na região $z$ infinito formam a álgebra de Virasoro. Deste modo concluímos que, a teoria do campo escalar $\phi$ descrita pela ação (2.161) é conforme se for considerada no horizonte (realmente numa vizinhança muito próxima do horizonte). As transformações conforme são geradas pelas cargas

$$
T[\xi]=\int_{-L / 2}^{L / 2} d z T_{++} \xi(z)
$$

onde

$$
\begin{aligned}
& T_{++}=T_{00}+T_{0 z} \\
& =\frac{1}{4}\left(\partial_{t} \phi+\partial_{z} \phi\right)^{2}-\frac{1}{4} q \Phi_{h}\left(\partial_{z}\left(\partial_{z}+\partial_{t}\right) \phi-\frac{1}{\beta_{H}}\left(\partial_{z}+\partial_{t}\right) \phi\right)
\end{aligned}
$$

Uma solução geral da eq.(2.169) é a soma de ondas planas que se movem para a direita e esquerda $\phi=\phi_{+}(t+z)+\phi_{-}(t-z)$. Contudo, só as ondas que se movem para a direita contribuem para $T_{++}$.

Para um valor grande de $z$ podemos usar a invariância de translação $z \rightarrow z+Z,(Z=$ const $)$ com o objetivo de ajustar $z$ no intervalo $-\frac{L}{2} \leq z \leq \frac{L}{2}$. Depois podemos tomar $L$ que tende a infinito. Considerando o campo $\phi$ neste intervalo assumimos condições de fronteira periódicas.

A partir de (2.161) encontramos a álgebra de Poisson

$$
\left\{\phi(z, t), \partial_{t} \phi\left(z^{\prime}, t\right)\right\}=\delta\left(z-z^{\prime}\right)
$$


e, como consequência, obtemos

$$
\left\{\left(\partial_{t}+\partial_{z}\right) \phi(z, t),\left(\partial_{t}+\partial_{z}\right) \phi\left(z^{\prime}, t\right)\right\}=\partial_{z} \delta\left(z-z^{\prime}\right)-\partial_{z^{\prime}} \delta\left(z-z^{\prime}\right) .
$$

Então a álgebra de Poisson das cargas (2.173) é

$$
\left\{T\left[\xi_{1}\right], T\left[\xi_{2}\right]\right\}=T\left[\left[\xi_{1}, \xi_{2}\right]\right]+\left(\frac{q \Phi_{h}}{4}\right)^{2} \int_{-L / 2}^{L / 2} C\left[\xi_{1}, \xi_{2}\right] d z
$$

onde $\left[\xi_{1}, \xi_{2}\right]=\xi_{1} \xi_{2}^{\prime}-\xi_{2} \xi_{1}^{\prime} \mathrm{e}$

$$
C\left[\xi_{1}, \xi_{2}\right]=\left(\xi_{1}^{\prime}+\beta_{H}^{-1} \xi_{1}\right)\left(\xi_{2}^{\prime}+\beta_{H}^{-1} \xi_{2}\right)^{\prime}-\left(\xi_{1}^{\prime}+\beta_{H}^{-1} \xi_{1}\right)^{\prime}\left(\xi_{2}^{\prime}+\beta_{H}^{-1} \xi_{2}\right)
$$

É fácil mostrar que (2.175) é idêntica à álgebra de Virasoro (2.143) se $\xi(z)$ é expandida em series de Fourier $\xi_{n}=e^{\frac{2 \pi}{L} n z}$ introduzindo os geradores de Virasoro

$$
L_{n}=\frac{L}{2 \pi} \int_{-L / 2}^{L / 2} d z e^{2 \frac{2 \pi}{L} n z} T_{++}
$$

que formam a álgebra

$$
i\left\{L_{k}, L_{n}\right\}=(k-n) L_{n+k}+\frac{c}{12} k\left(k^{2}+\left(\frac{L}{2 \pi \beta_{H}}\right)^{2}\right) \delta_{n+k, 0}
$$

com carga central $c=3 \pi q^{2} \Phi_{h}^{2}$.

\subsubsection{Configuração do modo zero e contagem dos es- tados}

Com o objetivo de usar a fórmula de Cardy (2.146) e contar o número de estados precisamos conhecer o valor de $L_{0}$. Este é determinado pela configuração do modo zero, que de fato é a configuração clássica. Em nosso caso a configuração clássica é $\phi=\phi_{h}$. Mas $L_{0}$ se anula nesse caso. Para resolver este problema note-se que é possível encontrar uma configuração mais geral perto do horizonte,

$$
\phi_{0}=\alpha+P z
$$

que obviamente é solução das equações de movimento.

Consideremos o sistema numa caixa, com período $L$. Primeiramente levamos em conta a função no intervalo $0 \leq z \leq L / 2$ e então estendemos por 
continuidade ao intervalo $-L / 2 \leq z \leq 0$, já que $\phi_{0}(-z)=\phi(z)$. A condição para a função $\phi_{0}$ é que se converte em $\phi_{h}$ no final do intervalo,

$$
\left.\phi_{0}\right|_{z=\frac{L}{2}}=\phi_{h}
$$

No ponto $z=0$ impomos a condição

$$
\left.\left(\partial_{z} \phi_{0}+\beta_{H}^{-1} \phi_{0}\right)\right|_{z=0}=0 \quad .
$$

Ambas as condições resultam na seguinte forma da configuração (2.178)

$$
\phi_{0}=2 \phi_{h}\left(\frac{z-\beta_{H}}{L-2 \beta_{H}}\right),
$$

no intervalo $0 \leq z \leq L / 2$, e obtemos $P=\partial_{z} \phi_{0}=\frac{2 \phi_{h}}{L}$.

Calculando $L_{0}$ obtemos

$$
L_{0}=\frac{L}{2 \pi} \int_{-L / 2}^{L / 2} d z T_{++}=\frac{L^{2} P^{2}}{8 \pi}=\frac{\phi_{h}^{2}}{2 \pi}=\frac{\Phi_{h}^{2}}{2 \pi q^{2}} .
$$

Aplicando a fórmula de Cardy obtemos

$$
S_{\text {conf }}=\pi \Phi_{h}^{2}=S_{B H}=\frac{\pi r_{h}^{2}}{G},
$$

Portanto a fórmula de Bekenstein-Hawking foi obtida a partir de considerações gerais da Relatividade Geral e da teoria conforme.

\subsection{Método das D-branas}

A teoria das cordas[47] conseguiu chamar a atenção nos últimos anos como uma teoria de unificação devido aos desdobramentos que teve desde seu surgimento. Mas a teoria de cordas é uma teoria quântica da gravidade e portanto é natural esperar que esta teoria dê uma explicação sobre quais são os graus de liberdade do buraco negro que justificam a entropia de BekensteinHawking.

No ano de 1996 Strominger e Vafa [51] conseguiram obter microscopicamente a entropia de um buraco negro no contexto da teoria de cordas. Eles utilizaram a teoria das D-branas $[48,49]$ para construir a solução do buraco negro e fazer a contagem dos graus de liberdade. 


\subsubsection{Solução de brana-negra na teoria de supercorda Tipo IIB}

Os buracos negros na teoria de cordas surgem como soluções da teoria de supergravidade (que é seu limite de baixas energias) correspondente. Estudar a ação de baixas energias da teoria de cordas é natural devido ao fato de que as partículas sem massa estão separadas por um enorme gap de massa $1 / \sqrt{\alpha^{\prime}}$ dos estados com massa.

A teoria de cordas existe em 10 dimensões, e portanto para obter buracos negros em 4 ou 5 dimensões temos que compactificar algumas dimensões. Então a ideia é construir configurações com branas enroladas que se intersectam e que depois de fazer uma redução dimensional levam a um buraco negro. Branas enroladas em dimensões compactas são vistas como objetos pontuais em um espaço $d$-dimensional.

Aqui mostraremos, particularmente, um buraco negro pentadimensional solução da teoria de cordas tipo IIB compactificada em $T^{5}=T^{4} \times S^{1}$, onde $T^{5}$ é um torus em 5 dimensões e $S^{1}$ representa um círculo. O sistema é composto por uma coleção de 1-branas e 5-branas enroladas no espaço compacto. As 1-branas enrola-se ao redor de $S^{1}$, que tem raio $R$. Entretanto as 5-branas enrolam-se no torus $T^{5}$. O número de 1-branas é $Q_{1}$ e o número de 5 -branas é $Q_{5}$. Também temos cordas abertas, sem massa, que portam momentum $P=$ $N / R$ na direção $S^{1}$. Cada uma destas cordas conectam uma 1-brana a uma 5 -brana. No total são $4 Q_{1} Q_{5}$ cordas bosônicas e o mesmo número de cordas fermiônicas. Mais adiante deixaremos claro como fazer esta construção.

Como já falamos anteriormente, esta configuração pode ser obtida a partir da teoria de supergravidade tipo $I I B$. Essa teoria possui $N=2$ supersimetrias em $D=10$. A teoria é chiral porque seus dois espinores Majorana-Weyl tem a mesma chiralidade.

Nesta teoria temos dois sectores dos modos sem massa: NS-NS (NS: Neveu-Schwarz) e R-R (R: Ramond). No setor NS-NS estão a métrica $G_{\mu \nu}$, a dois-forma $B_{\mu \nu}$ e o dilaton $\phi$. No setor R-R estão a zero forma $\chi$, a doisforma $A_{\mu \nu}$ e a quatro-forma $A_{\mu \nu \rho \delta}{ }^{2}$. O termo cinético padrão para estes dois últimos campos é [22]

$$
S \approx \int_{D} F_{n+1} \wedge * F_{n+1}
$$

\footnotetext{
${ }^{2} \mathrm{O}$ tensor de campos correspondente é autodual $F=d A=* F$. Devido a isto não é possivel escrever uma ação covariante correspondente.
} 
onde a integral é sobre o espaço D-dimensional, e $F_{n+1}=d A_{n}$.

Agora mostraremos quais são os análogos das fontes pontuais para uma ação como a anterior. Em geral, as cargas elétricas acoplam-se minimamente ao campo gauge $A_{n}$, e tal acoplamento é descrito pelo termo

$$
\int_{D} A_{n} \wedge * j_{n}
$$

onde a corrente elétrica $j_{n}$ é uma $n$ forma. A equação de Euler-Lagrange correspondente às ações (2.184) e (2.185) combinadas é

$$
d * F_{n+1}=* j_{n}
$$

Análogos às fontes pontuais são achados se a corrente se localiza numa superfície $(p=n-1)$ dimensional; neste caso temos

$$
\int_{D} A_{p+1} \wedge * j_{p+1}=\int_{p+1} A_{p+1}
$$

Portanto as fontes são membranas $p$-dimensionais, ou seja $p$-branas. Consideremos o mais simples exemplo onde o espaço-tempo é chato e a fonte é o plano $p$-dimensional $x^{i}=0$ para $i=p+1, \ldots, D-1$. É conveniente introduzir coordenadas esféricas nas dimensões transversais à brana, $x^{i}=\left(r, \phi^{1}, \ldots \ldots \phi^{D-p-2}\right)$. Então as equações de Maxwell generalizadas se reduzem a

$$
\triangle^{T} A_{01 . . p}(r) \simeq \delta(r)
$$

onde $\triangle^{T}$ e o operador de Laplace com respeito às coordenadas transversais e os índices $0,1, \ldots p$ são as direções paralelas à brana. A partir de (2.188) podemos encontrar o campo de calibre e o tensor de campo,

$$
A_{01 \ldots p} \simeq \frac{Q}{r^{D-p-3}} \quad \text { e } \quad F_{0 r 1 . . p} \simeq \frac{Q}{r^{D-p-2}}
$$

O parâmetro $Q$ é a carga elétrica. Como na eletrodinâmica podemos escrever a carga como uma integral de superfície

$$
Q \simeq \int_{D-p-2} * F_{p+2}
$$

onde a integração é sobre uma superfície $(D-p-2)$ dimensional que cobre a fonte. 
As fontes magnéticas são achadas intercambiando as funções da equação de movimento e a identidade de Bianchi. O potencial magnético $\tilde{A}$ satisfaz $d \tilde{A}=* F$. As fontes localizadas são $\tilde{p}$-branas $\operatorname{com} \tilde{p}=D-p-4$,

$$
\tilde{A}_{01 \ldots \tilde{p}} \simeq \frac{P}{r^{p+1}} \quad e \quad * F_{0 r 1 . . p} \quad \simeq \frac{Q}{r^{p+2}}
$$

A carga magnética é

$$
P \simeq \oint_{p+2} F_{p+2}
$$

Genericamente, as fontes elétrica e magnética têm diferentes dimensões, $p \neq \tilde{p}$ e têm que satisfazer à quantização de Dirac generalizada,

$$
P Q \simeq n \quad, \operatorname{com} \quad n \in \mathbf{Z} .
$$

Aqui nos estendemos em algumas características dos campos no setor $R-R$ devido à sua importância nos cenários com D-branas.

Então, levando-se em conta os dois setores mencionados anteriormente a ação de supergravidade IIB em $d=10$, no referencial de cordas é

$$
S=\frac{1}{16 \pi G_{10}} \int d^{10} x \sqrt{-G}\left[e^{-2 \phi}\left(R+4(\nabla \phi)^{2}-\frac{1}{3} H^{2}\right)-\frac{1}{2(p+2) !} F_{p+2}^{2}\right],
$$

onde $H=d B, F_{p+2}=d A_{p+1}$ são os tensores de campo associados com as formas. Todos os campos gauge serão considerados triviais exceto a doisforma $A_{\mu \nu}$ do sector R-R.

A seguir mostramos uma solução particular das equações de movimento correspondente a esta ação e que representa a configuração apresentada anteriormente [53],

$$
\begin{aligned}
d s_{(10)}^{2} & =f_{1}^{-\frac{1}{2}} f_{5}^{-\frac{1}{2}}\left(-d t^{2}+d x_{9}^{2}+k\left(d t-d x_{9}\right)^{2}\right)+ \\
& +f_{1}^{\frac{1}{2}} f_{5}^{\frac{1}{2}}\left(d x_{1}^{2}+\ldots+d x_{4}^{2}\right)+f_{1}^{\frac{1}{2}} f_{5}^{-\frac{1}{2}}\left(d x_{5}^{2}+\ldots . . d x_{8}^{2}\right), \\
e^{-2 \phi_{10}} & =f_{5} f_{1}^{-1} \\
A_{09} & =\frac{1}{2}\left(f_{1}^{-1}-1\right), \\
F_{i j k} & =(d A)_{i j k}=\frac{1}{2} \epsilon_{i j k l} \partial_{l} f_{5},
\end{aligned}
$$

onde

$$
f_{1}=1+\frac{\hat{Q}_{1}}{x^{2}}, \quad f_{5}=1+\frac{\hat{Q}_{5}}{x^{2}}, \quad k=\frac{\hat{N}}{x^{2}}
$$


com $x^{2}=x_{1}^{2}+\ldots+x_{4}^{2} \cdot \hat{Q}_{1}=c_{1}^{(5)} Q_{1}$ e $\hat{Q}_{5}=c_{5}^{(5)} Q_{5}$ são cargas pentadimensionais dos campos de calibre obtidas pela redução dimensional da R-R duas forma. As cargas correspondentes em 10 dimensões são as cargas elétrica e magnética da duas-forma. Os coeficientes $c_{1}^{(5)}$ e $c_{5}^{(5)}$ são as cargas unitárias correspondentes. Portanto $Q_{1}$ conta o número de D1-branas e $Q_{5}$ o número de D5-branas. A terceira carga $\hat{N}=c_{P}^{(5)} N$ corresponde ao momentum total na direção $\hat{9}$. As expressões dos coeficientes são

$$
c_{1}^{(5)}=\frac{4 G_{N}^{5} R_{9}}{\pi \alpha g}, \quad c_{5}^{(5)}=g \alpha, \quad c_{P}^{(5)}=\frac{4 G_{N}^{5}}{\pi R_{9}} .
$$

Agora, reduzimos dimensionalmente a métrica em (2.195) para obter a solução de buraco negro. Primeiramente são eliminadas as quatro dimensões transversais $x_{5}, \ldots x_{8}$,

$$
d s_{6}=f_{1}^{-\frac{1}{2}} f_{5}^{-\frac{1}{2}}\left(-d t^{2}+d x_{9}^{2}+k\left(d t-d x_{9}\right)^{2}\right)+f_{1}^{\frac{1}{2}} f_{5}^{\frac{1}{2}}\left(d x_{1}^{2}+\ldots+d x_{4}^{2}\right) .
$$

Neste processo, o dilaton muda como

$$
e^{-2 \phi_{6}}=e^{-2 \phi_{10}} \sqrt{G_{i n t}}=f_{5} f_{1}^{-1} \sqrt{f_{1}^{2} f_{5}^{-2}}=1,
$$

onde $G_{\text {int }}$ é o determinante das componentes da métrica correspondentes as dimensões compactificadas.

Ainda a dimensão $\hat{9}$ tem que ser compactificada. Usando a fórmula

$$
\tilde{G}_{00}=G_{00}-G_{09}^{2} / G_{99},
$$

reduzimos a direção $\hat{9}$ e achamos

$$
d s_{5 s t r i n g}^{2}=-f_{1}^{-\frac{1}{2}} f_{5}^{-\frac{1}{2}}(1+k)^{-1} d t^{2}+f_{1}^{\frac{1}{2}} f_{5}^{\frac{1}{2}}\left(d r^{2}+r^{2} d \Omega_{3}^{2}\right) .
$$

O dilaton agora fica como

$$
e^{-2 \phi_{5}}=e^{-2 \phi_{6}} \sqrt{f_{1}^{-\frac{1}{2}} f_{5}^{-\frac{1}{2}}(1+k)}=\frac{(1+k)^{\frac{1}{2}}}{f_{1}^{\frac{1}{4}} f_{5}^{\frac{1}{4}}} .
$$

Levando-se em conta a relação $g_{E}^{(5)}=e^{-4 \phi_{5} / 3} G_{s t r i n g}^{(5)}$, a métrica, no referencial de Einstein, fica 


$$
d s_{E}^{2}=-\frac{1}{\left(f_{1} f_{5}(1+k)\right)^{\frac{2}{3}}} d t^{2}+\left(f_{1} f_{5}(1+k)\right)^{\frac{1}{3}}\left(d x_{1}^{2}+\ldots .+d x_{4}^{2}\right)
$$

o que descreve um buraco negro pentadimensional supersimétrico, extremo, carregado. O horizonte localiza-se em $r=0$. A área do horizonte é

$$
A_{5}=\left.\left[r^{2}\left(f_{1} f_{5}(1+k)\right)^{1 / 3}\right]^{3 / 2}\right|_{r=0}\left(2 \pi^{2}\right)=\left(2 \pi^{2}\right) \sqrt{c_{1}^{(5)} c_{5}^{(5)} c_{P}^{(5)} N Q_{1} Q_{5}} .
$$

Levando-se em conta a fórmula de Bekenstein-Hawking e usando as relações (2.197) encontramos a entropia

$$
S=\frac{A_{5}}{4 G_{N}^{(5)}}=2 \pi \sqrt{N Q_{1} Q_{5}}
$$

\subsubsection{Número de estados microscópicos.}

Nesta seção mostramos a contagem dos graus de liberdade correspondentes ao buraco negro apresentado na seção anterior. Apesar de haver na literatura contagens mais sofisticadas [51], [54, 55] aqui apresentamos um modo mais simples de se obter a entropia[52].

Primeiramente vemos um pouco melhor o sistema de branas $D 1-D 5$. Neste sistema temos várias cordas abertas a considerar: aquelas que vão de uma $D 1$-brana para outra $D 1$-brana, que denotamos como corda $(1,1)$, também temos as cordas $(5,5)$. $(1,5)$ e $(5,1)$ (as duas últimas sendo diferentes porque as cordas são orientadas). As cordas $(1,5),(5,1)$ são sem massa enquanto as cordas $(1,1)$ e $(5,5)$ são massivas e não são consideradas para a contagem porque violam a condição BPS.

Então consideramos as cordas $(1,5)$ e $(5,1)$. Temos 2 bósons com condições de fronteira tipo NN, 4 com ND e 4 com DD (Ver Apêndice D.2). A energia de vácuo dos bósons é então $E=4(-1 / 24+1 / 48)$. Os fermions do sector NS tem energia do vácuo $E=4(1 / 24-1 / 48)$ que cancela exatamente a contribuição bosônica. Este vácuo é um espinor sobre $S O(4)_{5678}$ e obedece a condição GSO de chiralidade $\Gamma^{5} \Gamma^{6} \Gamma^{7} \Gamma^{8} \chi=\chi$. Portanto fica uma representação bidimensional. Mas como temos duas possíveis orientações das cordas e elas podem fixar-se em quaisquer das diferentes branas presentes obtemos $4 Q_{1} Q_{5}$ bósons. No setor Ramond a energia do vácuo também é zero e por 
supersimetria também temos $4 Q_{1} Q_{5}$ fermions. Em termos gerais, a contagem é a mesma que para os modos oscilatórios (à esquerda) de $4 Q_{1} Q_{5}$ campos livres superconformes. A fórmula assintótica para a degenerescência do nível "N" numa teoria conforme com carga central $c$ é $d(N) \sim e^{2 \pi \sqrt{c N / 6}}$. Incluindo os fermions e os bosons, temos $c=4 Q_{1} Q_{4}\left(1+\frac{1}{2}\right)=6 Q_{1} Q_{5}$, o que nos leva

$$
S=2 \pi \sqrt{Q_{1} Q_{5} N}
$$

em perfeita concordância com a fórmula de Bekenstein-Hawking (2.205). 


\section{Capítulo 3}

\section{Limites para a Entropia.}

\subsection{Relação $S / E$.}

Classicamente, a entropia é uma medida do espaço de fase disponível do sistema estudado. Levando-se em conta este fato, não é difícil compreender porque deve existir um limite superior para a relação entre a entropia e a energia, $S / E$. Se considerarmos um sistema com energia não superior a $E$, teremos um limite do espaço dos momentos disponíveis. Se o sistema também for limitado no espaço, o espaço de fase também o será, bem como a entropia. Mas este simples argumento não leva à forma específica do limite para a relação $S / E$. De fato, parece impossível obter alguma expressão sem saber os detalhes do sistema. Contudo, no ano de 1981, Bekenstein [57], usando a física dos buracos negros, encontrou a forma específica para o limite superior de $S / E$ para sistemas com auto-gravidade desprezível. Fazendo uso da Segunda Lei Generalizada da Termodinâmica para um corpo absorvido por um buraco negro, Bekenstein encontrou o resultado

$$
S / E \leq 2 \pi R,
$$

onde $R$ e a dimensão do objeto.

Um aspecto interessante do argumento usado para determinar (3.1) é que se usa uma lei cujos fundamentos radicam na gravitação, para derivar o limite $S / E$ de um sistema com gravidade desprezível, um limite que não tem relação com a gravidade (não envolve $G$ ).

Uma questão que aparece é quão dependente é o limite (3.1) de se supor que o sistema tenha auto-gravidade desprezível. Para clarear consideramos 
um buraco negro de Kerr, que é um sistema com os máximos efeitos gravitacionais. Seja sua energia $E$; então sua area será

$$
A=4 \pi\left[E+\left(E^{2}-a^{2}-Q^{2}\right)^{1 / 2}\right]^{2}+a^{2}
$$

onde $a$ e o momento angular específico e $Q$ a carga. É claro que $R<2 E$, a igualdade correspondendo a $a=Q=0$. Considerando $S=A / 4$, é claro que o buraco de Kerr satisfaz (3.1); o buraco de Schwarzschild dá a igualdade.

Se sistemas com auto-gravidade desprezível e também buracos negros obedecem (3.1), é razoavel assumir que este limite também se cumpra para sistemas nos quais a gravidade tem uma força intermediaria entre os dois casos anteriores. Isto nos induz a pensar na universalidade deste limite. Devemos salientar que há uma polêmica sobre a universalidade do limite de Bekenstein. Discussões a respeito desta controvérsia podem ser encontradas em $[58,59,60],[61],[62],[63]$.

Com o objetivo de provar a relação (3.1) para um sistema com autogravidade fraca consideremos um corpo de massa $m$ que é jogado na direção radial de um buraco negro de Schwarzschild. É conhecido da Relatividade Geral que a energia $E_{p}$ de um objeto medido por um observador numa posição constante $r$ esta relacionada com a energia $E$ medida por um observador no infinito mediante o fator de deslocamento infravermelho

$$
E=\sqrt{-g_{t t}} E_{p}
$$

Neste caso $E_{p}=m$ onde $m$ é a massa de repouso do corpo.

A energia calculada no ponto $r=r_{h}+\epsilon$ é

$$
E=m\left(\frac{\epsilon}{r_{h}}\right)^{1 / 2}
$$

Com o intenção de encontrar a mudança na entropia do buraco negro causada pela absorção do corpo, devemos calcular $E$ no ponto de captura, a uma distância própria $R$ fora do horizonte,

$$
R=\int_{r_{h}}^{r_{h}+\epsilon(R)} \frac{d r}{\sqrt{1-\left(\frac{r_{h}}{r}\right)}}
$$

Integrando obtemos

$$
R=2 \sqrt{r_{h} \epsilon}
$$


portanto a energia pode ser reescrita como

$$
E=\frac{m R}{2 r_{h}}
$$

A assimilação do corpo resulta em uma variação $d M=E$ na massa do buraco negro. Usando a primeira lei da termodinâmica

$$
d M=T d S
$$

e a expressão da temperatura $T=1 / 4 \pi r_{h}$ obtemos que a entropia do buraco negro aumenta como

$$
(d S)_{b h}=2 \pi m R \text {. }
$$

Todavia, sabemos da Segunda Lei Generalizada(SLG), que a relação $(\Delta S)_{T} \equiv$ $(d S)_{b h}-S_{c o} \geq 0$ deve ser satisfeita. Isto implica em um limite superior para a entropia do corpo

$$
S_{c o} \leq 2 \pi E R \text {. }
$$

Este resultado não depende dos parâmetros do buraco negro, como era de se esperar.

\subsubsection{Relação S/E na gravidade de ordem superior}

Nesta seção mostraremos o comportamento da relação entropia e energia no caso da teoria presentada em 2.1.2. O mesmo procedimento da seção anterior será usado para se conseguir este objetivo. Portanto, primeiramente, trabalharemos com a solução de buraco negro (2.86).

Levando-se em conta a expressão para a entropia (2.98) e a definição do raio do horizonte obtemos

$$
S / E=\frac{\frac{2 \pi k}{G_{k}} \frac{r_{h}^{d-2 k}}{d-2 k}}{\frac{r_{h}^{d-2 k-1}}{2 G_{k}}}=\frac{4 \pi k r_{h}}{d-2 k}=\frac{2 k}{d-2 k}(S / E)_{B e k},
$$

onde $(S / E)_{B e k}=2 \pi r_{h}$. Este resultado constitui uma generalização do limite de Bekenstein para buracos negros na gravidade de Lovelock.

No caso de autogravidade desprezivel consideramos que o corpo seja jogado no buraco. As grandezas são calculadas do mesmo modo e obtemos

$$
E=m \gamma^{1 / 2}\left(\frac{\epsilon}{r_{h}}\right)^{1 / 2}
$$


Com a intenção de encontrar a mudança na entropia do buraco negro causada pela absorção do corpo, devemos calcular $E$ no ponto de captura, a uma distância própria $R$ fora do horizonte,

$$
R=\int_{r_{h}}^{r_{h}+\epsilon(R)} \frac{d r}{\sqrt{1-\left(\frac{r_{h}}{r}\right)^{\gamma}}} .
$$

Integrando, obtemos

$$
R=2 \sqrt{\frac{r_{h} \epsilon}{\gamma}} .
$$

Substituindo esta expressão em (3.12) a energia pode ser reescrita como

$$
E=\frac{m \gamma R}{2 r_{h}}
$$

Lembrando que a temperatura para este tipo de buraco negro é

$$
T=\frac{\gamma}{4 \pi r_{h}},
$$

e usando novamente a relação termodinâmica $d M=T d S$, obtemos o mesmo resultado, com a ajuda da (SLG),

$$
S_{c o} \leq 2 \pi E R
$$

Mais uma vez a universalidade do limite de Bekenstein para sistemas com autogravidade fraca fica provada. 


\subsection{Limites holográficos da entropia na cos- mologia.}

Da física dos buracos negros temos descoberto coisas surprendentes sobre a natureza do espaço tempo e sua relação com a matéria, energia e entropia. Portanto, é natural, dentro do contexto da gravidade, tentar estender esses conhecimentos na cosmologia. Nesta seção mostraremos em particular a primeira tentativa de aplicar a idéia de holografia na cosmologia.

\subsubsection{Universos Chatos.}

Primeiramente dedicaremos atenção para a cosmologia usual de RobertsonWalker num espaço chato. O elemento de linha toma a forma

$$
d s^{2}=-d t^{2}+a^{2}(t) d x^{i} d x^{i}
$$

Consideraremos que o espaço tempo é de $d+1$ dimensões, portanto o valor de $i$ vai de 1 a $d$.

A proposta de princípio holográfico para a Cosmologia, por parte de Fischler e Susskind, foi que o fluxo de entropia através de uma frente de onda de luz contraindo-se é menor ou igual que a área da superficie onde a onda de luz foi originada. No caso de um universo isotrópico isto se reduz a simples condição:

$A$ entropia contida dentro de um volume de raio $R_{H}$ (raio do horizonte) não deve exceder a área do horizonte, isto é,

$$
\sigma R_{H}^{d}<\left[a R_{H}\right]^{d-1}
$$

onde $\sigma$ é a densidade de entropia, $R_{H}$ é raio do horizonte, determinado pela expressão

$$
R_{H}(t)=\int_{0}^{t} \frac{d t^{\prime}}{a\left(t^{\prime}\right)} .
$$

Supondo-se que $a(t) \sim t^{\alpha}$ temos

$$
R_{H}(t) \sim t^{1-\alpha} .
$$

Deste modo para que (3.19) seja verdadeira no futuro remoto, deve-se satisfazer a

$$
\alpha>\frac{1}{d} \text {. }
$$


Isto implica que existe um limite inferior para a expansão.

Este limite pode ser transladado num limite da equação de estado. Tomase a forma usual desta equação

$$
P=\gamma \rho,
$$

onde $P$ e a pressão e $\rho$ a densidade de energia. Os métodos conhecidos nos levam a solução das equações de Einstein com o fator de escala dado por

$$
a(t) \sim t^{\frac{2}{d(1+\gamma)}} .
$$

Consequentemente $\alpha=\frac{2}{d(1+\gamma)}$ e a desigualdade (3.19) converte-se em

$$
\gamma<1
$$

Mas esta imposição também vem de considerações muito diferentes. Uma violação de (3.25) significaria que velocidade do som é maior que a da luz. Esta concordância pode ser uma evidência de que a proposta feita por Fischler e Susskind é acertada.

Outro exemplo envolve universos chatos porém anisotrópicos, com métrica

$$
d s^{2}=-d t^{2}+\sum_{i} t^{2 \alpha_{i}} d x_{i}^{2}
$$

Neste caso a relação $S / A$ seria

$$
S / A=\frac{\prod_{i} R_{H, i}}{\left[\prod_{j} t^{p_{j}} t^{1-p_{j}}\right]^{(d-1) / d}},
$$

onde $R_{H, i}=t^{1-\alpha}$ é o raio do horizonte na direção $i$. Da equação anterior obtemos

$$
S / A=t^{1-\sum_{i} \alpha_{i}} .
$$

Os expoentes satisfazem às seguintes condições (condições de Kasner), obtidas a partir das equações de Einstein,

$$
\begin{aligned}
& \sum_{i} \alpha_{i}=1, \\
& \sum_{i} \alpha_{i}^{2}=1 .
\end{aligned}
$$

Evidentemente, levando-se em conta (3.29), $S / A$ é constante no tempo para esses universos. Portanto, dependendo das condições iniciais o princípio holográfico poderia ser saturado por esses universos. 


\subsubsection{Universo Fechado e Inflação.}

Um universo de Friedman-Robertson-Walker (FRW) é caracterizado pela métrica

$$
d s^{2}=-d t^{2}+a^{2}(t)\left(d \chi^{2}+\sin ^{2} \chi d \Omega^{2}\right)
$$

Neste caso a área da esfera causal é

$$
A=4 \pi a^{2}(t) \sin ^{2} \chi_{H},
$$

enquanto o seu volume é dado por

$$
V=\int_{0}^{\chi_{H}} d \chi \sin ^{2} \chi d \Omega=\pi\left(2 \chi_{H}-\sin 2 \chi_{H}\right) .
$$

Portanto obtemos, para a relação entre entropia e área a expressão

$$
S / A=\sigma \frac{2 \chi_{H}-\sin 2 \chi_{H}}{2 a^{2}\left(\chi_{H}\right) \sin ^{2}\left(\chi_{H}\right)},
$$

onde $\sigma$ é a densidade da entropia. Agora consideremos o caso de um universo dominado por matéria escura fria, com $P \ll \rho$. Neste caso $a=$ $a_{\max } \sin ^{2}\left(\chi_{H} / 2\right)$. O momento $\chi_{H}=\pi$ corresponde à máxima expansão, $a=a_{\max }$, e obviamente o limite holográfico de $S / A$ é violado. Este resultado pode ser interpretado de duas formas: a solução de universos fechados deve ser excluida, ou, o princípio holográfico deve ser reformulado. Mas existem outros critérios onde isto não acontece. Por exemplo, na prescrição proposta por Bousso[65], o universo fechado satisfaz à holografia.

Também devemos comentar que, numa cosmologia inflacionária, o critério de FS só pode ser aplicado depois do período do "reaquecimento" devido ao fato deste processo ser altamente não adiabático e portanto não satisfaz a condição de adiabaticidade proposta por eles. Isto significa que a integração (3.20) não deve começar em $t=0$ mas em $t=t_{r e}$, depois do "reaquecimento".

\subsection{Exemplo}

Nesta seção mostraremos a aplicação do critério FS [67] para um modelo cosmológico no contexto de dimensões extras [68]. O estudo das dimensões extras despertou muita atenção nos últimos três anos como conseqüencia da proposta feita por N. Arkani-Hamed et al [69] para resolver o problema da 
hierarquia. Na física das altas energias o problema da hierarquia é conhecido como a enorme diferença de ordem de grandeza entre as duas escalas fundamentais de energia na natureza: a electrofraca $m_{E W} \sim 10^{3} \mathrm{GeV}$ e a escala de Planck $M_{P l}=G_{N}^{-1 / 2} \sim 10^{19} \mathrm{GeV}$. Para resolver este problema $\mathrm{N}$. Arkani-Hamed et al simplesmente assumiram que $m_{E W}$ é a única escala fundamental na natureza. Para compatibilizar esta proposta com o valor conhecido da escala de Planck foi considerada a existência de $n$ dimensões extras compactas com raio $\sim R$. Assim, a escala de Planck $M_{P l(4+n)}$ desta teoria $(4+n)$ dimensional é da ordem de $m_{E W}$ concordando com a filosofia adotada. Para determinar o valor das dimensões extras calcula-se o potencial de duas massas de teste $m_{1}, m_{2}$, primeiramente para uma distância muito menor que o raio das dimensões extra $(r \ll R)$. Aplicando a lei de Gauss em $(4+n)$ dimensões temos

$$
V(r) \sim \frac{m_{1} m_{2}}{M_{P l(4+n)}^{(n+2)}} \frac{1}{r^{n+1}} \quad(r \ll R) .
$$

Por outro lado, se as massas são colocadas a uma distância $(r \gg R)$, o fluxo das linhas gravitacionais não penetra nas dimensões extras fazendo com que o potencial seja

$$
V(r) \sim \frac{m_{1} m_{2}}{M_{P l(4+n)}^{(n+2)} R^{n}} \frac{1}{r} \quad(r \gg R) .
$$

Levando-se em conta que $M_{P l}^{2}=G_{N}^{-1}$, obtém-se a relação

$$
M_{P l}^{2} \sim M_{P l(4+n)}^{2+n} R^{n} .
$$

Colocando $M_{P l(4+n)} \sim m_{E W}$ e exigindo que $R$ reproduza o valor observado de $M_{P l}$ temos

$$
R \sim 10^{\frac{30}{n}-17} \mathrm{~cm} \times\left(\frac{T e v}{m_{E W}}\right)^{1+\frac{2}{n}} .
$$

No caso de uma dimensão extra $n=1, R \sim 10^{13} \mathrm{~cm}$ o que é impossível porque implica em desvios da gravidade de Newton para distâncias dentro do sistema solar. Entretanto para $n \geq 2$ as modificações à gravidade são notáveis a distâncias menores que as conferidas experimentalmente até agora $(1 \mathrm{~cm})$. Por exemplo, para $n=2(R \sim 100 \mu m-1 \mathrm{~mm})$. Isto é particularmente interessante devido ao fato de que novos experimentos serão feitos no futuro procurando desvios da gravidade padrão precisamente neste patamar de distâncias [70]. 
O fato de se considerar a possibilidade do espaço-tempo com mais de quatro dimensões é uma idéia recorrente nas teorias de unificação desde a proposta feita originalmente por Kaluza [71] e Klein [72].

No modelo que vamos apresentar, a dinâmica das dimensões é descrita pelas equações de Einstein. As dimensões são divididas em nosso mundo quadridimensional e em $n$ dimensões internas. Em termos concretos, no modelo cosmológico de N. Arkani-Hamed temos uma era primordial inflacionária (sem "reaquecimento") seguida por uma época onde as dimensões de nosso mundo sofrem uma contração enquanto as dimensões internas continuam se expandindo até seu valor de estabilidade. Este período de contração resulta muito interessante porque representa uma alternativa para a fase de reaquecimento dos modelos inflacionários padrões[75]. Depois, quando o período de contração termina, o universo vai assintoticamente para um comportamento tipo radiação descrito pelo modelo FRW. Nosso interesse é na fase de contração, precisamente devido ao fato de ser uma alternativa ao reaquecimento. Nos cálculos consideramos que existem duas dimensões extras $n=2$.

A ação total que descreve este universo esta formada pela parte volumétrica,

$$
S_{\text {vol }}=-\int d^{6} x \sqrt{-\operatorname{det} G_{(6)}}\left(M_{*}^{4} \mathcal{R}-\mathcal{L}_{\text {materia }}+\ldots\right),
$$

e a parte das quatro dimensões, nossa brana,

$$
S_{\text {brana }}=-\int d^{4} x \sqrt{-\operatorname{det} g_{(4)}}\left(\mathcal{L}_{\text {modelo padrão }}+\ldots\right),
$$

onde $\mathcal{L}_{\text {materia }}$ é a Langrangeana dos campos no volume $(D=6)$. Estes campos dão origem ao potencial de estabilização $V$. As ellipses denotam os termos com derivadas superiores dos campos.

A métrica de fundo consistente com as simetrias do sistema brana-volume é da forma

$$
g_{\mu \nu}=\left(\begin{array}{ccc}
-1 & & \\
& a(t)^{2} g_{I J} & \\
& & b(t)^{2} g_{i j}
\end{array}\right)
$$

onde $a(t)$ é o fator de escala correspondente às dimensões físicas (observáveis ) tri-dimensionais, e $b(t)$, corresponde às dimensões internas. 
Portanto considerando o potencial de estabilização das dimensões internas $V(b)$ assim como materia na brana quadridimensional obtemos as seguintes equações [68]

$$
\begin{aligned}
& 6 H_{a}^{2}+2 H_{b}^{2}+12 H_{a} H_{b}=\frac{V+\rho}{M_{*}^{4} b^{2}}, \\
& \frac{\ddot{b}}{b}+H_{b}^{2}+3 H_{b} H_{a}=\frac{1}{M_{*}^{4} b^{2}}\left(\frac{V}{2}-\frac{b}{8} \frac{\partial V}{\partial b}+\frac{\rho-3 P}{8}\right), \\
& \frac{\ddot{a}}{a}+2 H_{a}^{2}+2 H_{b} H_{a}=\frac{1}{M_{*}^{4} b^{2}}\left(\frac{b}{8} \frac{\partial V}{\partial b}+\frac{\rho+P}{8}\right), \\
& \dot{\rho}+3 H_{a}(P+\rho)=0 .
\end{aligned}
$$

onde $H_{a} \equiv \dot{a} / a$ e $H_{b} \equiv \dot{b} / b$ são os parâmetros de Hubble, $a$ e $b$ os fatores de escala, e o ponto denota a derivada com respeito a $t$. Nas equações acima $M_{*}^{4}=M_{P l}^{2} /\left(b_{0}\right)^{2}$ onde $b_{0}$ é o valor da coordenada interna estabilizada, $\rho$ é a densidade de energia na brana e $P$ a pressão. Essas grandezas estão no referencial de corda. A razão é que a cinemática neste referencial é expressa automaticamente em termos de unidades medidas por observadores que estão na brana 3-dimensional. Usando os dados de COBE [76] pode ser verificado que os valores de $\rho$ e $P$ são menores que os do potencial $V$. Portanto, com o objetivo de simplificar, os valores da densidade de energia e a pressão são desprezados no cálculo. Também supõe-se que o potencial tem a forma $V=W b^{-p}$, onde $W$ é um parâmetro.

Com o objetivo de mostrar características genéricas das soluções consideraremos o caso limite onde o potencial é desprezado. A evolução dos fatores de escala é controlada completamente pela energia cinética, então temos as equações de Einstein no vácuo

$$
R_{\mu \nu}=0 .
$$

As soluções $(4+n)$ dimensionais têm a forma

$$
d s^{2}=-d t^{2}+a_{i}^{2}\left(\frac{t}{t_{i}}\right)^{2 k} d x_{3}^{2}+b_{i}^{2}\left(\frac{t}{t_{i}}\right)^{2 l} d y_{n}^{2},
$$

onde $a_{i}$ e $b_{i}$ são os valores iniciais dos fatores de escala. Relembrando os resultados da seção 3.2.1 já sabemos que as potências $k$ e $l$ são determinadas pela as equações de Einstein, e obtemos

$$
3 k+2 l=1,
$$




$$
3 k^{2}+2 l^{2}=1
$$

As soluções destas equações são

$$
\begin{aligned}
& k=\frac{3 \mp \sqrt{24}}{15}, \\
& l=\frac{2 \pm \sqrt{24}}{10} .
\end{aligned}
$$

Fenomenologicamente, precisamos que as dimensões internas aumentem e alcancem seu valor de estabilização. Para tal, devemos escolher o sinal superior em cada uma das equações (3.46). Portanto o valor das potências são $k=-0.1266$ e $l=0.69$.

Para o caso geral onde consideramos o potencial $V$ as equações de Einstein (3.41) tem soluções exatas, contudo faremos algums comentários gerais antes. Notemos que para certo patamar do parâmetro $p$ as soluções exatas devem convergir as soluções tipo Kasner (potencial zero). Substituindo as potências de Kasner nas equações de Einstein temos que esse patamar pode ser encontrado para o caso em que o membro direito se anula mais rapidamente que o membro esquerdo conforme o tempo passa. Sabendo-se que o membro esquerdo sempre cai com $t^{-2}$ temos

$$
(2+p) 0.69>2
$$

e assim obtemos um valor crítico de $p_{c r}=0.899$.

Para solucionar o sistema (3.41), definimos

$$
a=a_{i} e^{\alpha(t)} \quad, b=b_{i} e^{\beta(t)},
$$

onde $a_{i}$ e $b_{i}$ são os valores iniciais dos fatores de escala depois de finalizada a epoca de inflação. Além disto, definindo o parâmetro $\omega=\frac{W}{M_{*}^{4} b_{i}^{2+p}}$, substituindo (3.48) em (3.41), e passando a uma nova variável temporal

$$
d \tau=-e^{-3 \alpha-2 \beta} d t
$$

chega-se, depois de um pouco de álgebra, às equações de movimento em uma forma mais favoravel para sua análise explicita,

$$
\begin{gathered}
6 \alpha^{\prime 2}+2 \beta^{\prime 2}+12 \alpha^{\prime} \beta^{\prime}=\omega e^{6 \alpha+(2-p) \beta}, \\
\beta^{\prime \prime}=\frac{(4+p) \omega}{8} e^{6 \alpha+(2-p) \beta},
\end{gathered}
$$




$$
\alpha^{\prime \prime}=-\frac{p \omega}{8} e^{6 \alpha+(2-p) \beta},
$$

onde ' denota as derivadas com respeito a $\tau$. A forma específica de $\tau=\tau(t)$ pode ser determinada depois de se achar as soluções para $\alpha$ e $\beta$.

As equações (3.52) são integráveis. Considerando a combinação linear $4 \alpha+\frac{4 p}{4+p} \beta$ e usando as equações diferenciais de segunda ordem do sistema podemos verificar que

$$
4 \alpha^{\prime \prime}+\frac{4 p}{4+p} \beta^{\prime \prime}=0
$$

cuja solução é

$$
4 \alpha+\frac{4 p}{4+p} \beta=C_{1}+C_{2} \tau
$$

onde $C_{1}$ e $C_{2}$ são constantes de integração que serão determinadas depois.

Para se achar a outra integral de movimento definimos a variável

$$
X=6 \alpha+(2-p) \beta .
$$

Então a outra equação se converte em

$$
X^{\prime \prime}=\frac{\Delta}{8} \omega e^{X},
$$

onde $\Delta=8-8 p-p^{2}$. Integrando obtém-se

$$
X^{\prime 2}=2 \frac{\Delta}{8} \omega e^{X}+E_{0}
$$

A constante $E_{0}$ pode ser determinada se substituimos (3.57) e a primeira derivada de (3.54) na primeira equação do sistema (3.52), portanto

$$
E_{0}=\frac{3(4+p)^{2}}{32} C_{2}^{2}
$$

Então o sistema (3.52) foi reduzido a um vínculo funcional (3.54) e a uma equação simples de primeira ordem,

$$
X^{\prime 2}=\frac{\Delta}{4} \omega e^{X}+\frac{3(4+p)^{2}}{32} C_{2}^{2} .
$$

As soluções do sistema possuem forma diferente dependendo do valor de $C_{2}$ e $\Delta$. As soluções podem ser classificadas em quatro tipos:
(1) $\Delta=0$
(2) $\Delta>0, C_{2}=0$
(3) $\Delta>0, C_{2}<0$;
(4) $\Delta<0, C_{2}<0$. 
$\mathrm{O}$ valor crítico $\Delta=0$ corresponde a $p \simeq 0.899$. Neste caso a solução é

$$
\begin{gathered}
a=a_{i}\left(\frac{t}{t_{i}}\right)^{k}, \\
b=b_{i}\left(\frac{t}{t_{i}}\right)^{l},
\end{gathered}
$$

com $k=-0.1266$ e $l=0.69$. Estes valores satisfazem às duas equações algebricas

$$
3 k+2 l=1,
$$

e

$$
3 k^{2}+2 l^{2}=1 .
$$

Portanto esta solução descreve um universo tipo Kasner.

Aplicando o critério F-S para universos anisotrópicos, a seguinte relação entropia/área é obtida, estando as grandezas com barra definidas no referencial de Einstein,

$$
S / A=\frac{\bar{\sigma}\left(\bar{R}_{H, \bar{a}}\right)^{3}\left(\bar{R}_{H, \bar{b}}\right)^{2}}{\left[\left(\bar{a} \bar{R}_{H, \bar{a}}\right)^{3}\left(\bar{b} \bar{R}_{H, \bar{b}}\right)^{2}\right]^{4 / 5}} .
$$

Com o objetivo de se calcular a relação $S / A(3.65)$ usamos a expressão que relaciona a métrica no referencial de Einstein com a métrica no referencial de corda [68]

$$
\bar{g}_{\mu \nu}=b^{2} g_{\mu \nu} .
$$

Usando este mapa, obtemos

$$
\bar{a}=b a \quad \bar{b}=b b \quad d \bar{t}=b d t .
$$

Portanto,

$$
\bar{R}_{H, \bar{a}}=R_{H, a} \quad \bar{R}_{H, \bar{b}}=R_{H, b} .
$$

Agora, a relação $S / A$ pode ser reescrita como

$$
S / A=\frac{1}{b^{4}} \frac{\sigma\left(R_{H, a}\right)^{3}\left(R_{H, b}\right)^{2}}{\left[\left(a R_{H, a}\right)^{3}\left(b R_{H, b}\right)^{2}\right]^{4 / 5}} .
$$

Como estamos trabalhando com um modelo inflacionário sem o "reaquecimento" podemos considerar a evolução do universo depois da fase de "de Sitter", que é o início da era de contração. 
Levando-se em conta os fatores de escala (3.61) e (3.62) obtemos,

$$
S / A=\frac{\sigma}{a_{i}^{3} b_{i}^{6}} c_{1} t_{i} f(x),
$$

onde

$$
c_{1}=\frac{1}{(1-k)^{3 / 5}} \frac{1}{(1-l)^{2 / 5}}=1,4873
$$

e

$$
f(x)=\frac{1}{x^{4 l}} \frac{\left(x^{1-k}-1\right)^{3}\left(x^{1-l}-1\right)^{2}}{\left(x-x^{k}\right)^{12 / 5}\left(x-x^{l}\right)^{8 / 5}},
$$

onde $x=t / t_{i}$.

A forma de $f(x)$ é mostrada na figura 3.1. Usando (3.63) pode-se conferir que o valor assintótico de $f(x)$ é zero. Portanto, se o limite holográfico for satisfeito quando $f(x)$ alcança seu valor máximo, posteriormente também o será.

As soluções no caso $\Delta>0(0 \leq p<0.899)$ e $C_{2}=0$ podem ser calculadas de modo simples. A equação (3.59) simplifica e fica sendo

$$
X^{\prime}=\sqrt{\frac{\Delta}{4} \omega} \exp (X / 2) .
$$

Integrando temos

$$
\exp (X)=\frac{16}{\Delta \omega} \frac{1}{\tau^{2}} .
$$

Usando (3.54) podemos determinar as soluções para $\alpha$ e $\beta$

$$
\begin{gathered}
\alpha=\frac{(4+p)(2-p) C_{1}}{4 \Delta}-\frac{p}{\Delta} X, \\
\beta=\frac{4+p}{\Delta} X-\frac{3(4+p) C_{1}}{2 \Delta} .
\end{gathered}
$$

Portanto obtemos

$$
\begin{aligned}
e^{\alpha} & =\exp \left(\frac{(4+p)(2-p) C_{1}}{4 \Delta}\right)\left(\frac{\Delta \omega}{16}\right)^{\frac{p}{\Delta}} \tau^{\frac{2 p}{\Delta}} \\
e^{\beta} & =\exp \left(-\frac{3(4+p) C_{1}}{2 \Delta}\right)\left(\frac{16}{\Delta \omega}\right)^{\frac{4+p}{\Delta}} \frac{1}{\tau^{\frac{8+2 p}{\Delta}}}
\end{aligned}
$$


Agora, podemos usar (3.49) para colocar (3.77) em função de $t$. Integrando obtemos

$$
\tau \sim t^{-\frac{\Delta}{(2+p)(4+p)}} .
$$

Depois de fazer os reescalonamentos corretos encontramos finalmente os fatores de escala

$$
\begin{aligned}
& a=a_{i}\left(\frac{t}{t_{i}}\right)^{-\frac{2 p}{(2+p)(4+p)}} \\
& b=b_{i}\left(\frac{t}{t_{i}}\right)^{\frac{2}{2+p}}
\end{aligned}
$$

Portanto, as potências são

$$
\begin{gathered}
v=-\frac{2 p}{(2+p)(4+p)}, \\
u=\frac{2}{2+p}
\end{gathered}
$$

e satisfazem à relação

$$
3 v+2 u>1 .
$$

Repetindo o mesmo procedimento que no caso anterior encontramos

$$
S / A=\frac{\sigma}{a_{i}^{3} b_{i}^{6}} c_{2} t_{i} g(x)
$$

onde para $p<0.899$

$$
\begin{gathered}
c_{2}=\frac{1}{(1-v)^{3 / 5}} \frac{1}{(1-u)^{2 / 5}}, \\
g(x)=\frac{1}{x^{4 u}} \frac{\left(x^{1-v}-1\right)^{3}\left(x^{1-u}-1\right)^{2}}{\left(x-x^{v}\right)^{12 / 5}\left(x-x^{u}\right)^{8 / 5}},
\end{gathered}
$$

e para $p=0$,

$$
\begin{gathered}
c_{2}=1 \\
g(x)=\frac{1}{x^{4}} \frac{(x-1)^{3 / 5}(\ln (x))^{2 / 5}}{x^{8 / 5}} .
\end{gathered}
$$

Na figura 3.2 a função $g(x)$ é mostrada para valores diferentes do parâmetro $p(0,0.5,0.7)$. Também neste caso o valor assintótico de $g(x)$ é zero. Na figura 3.3 colocamos uma comparação entre as funções $f(x)$ e $g(x)$. 
Para resolver a equação (3.59) no caso $\left(\Delta>0, C_{2}<0\right)$ fazemos a substituição

$$
e^{-X}=\frac{8 \Delta \omega}{3(4+p)^{2} C_{2}^{2}} \sinh ^{2}(\theta)
$$

então a equação (3.59) se reduz à equação diferencial $\theta^{\prime}=\left(\frac{3(4+p)^{2}}{128}\right)^{1 / 2} C_{2}$ cuja solução é $\theta=\left(\frac{3(4+p)^{2}}{128}\right)^{1 / 2} C_{2} \tau$. Portanto, a solução de (3.59) é

$$
e^{-X}=\frac{8 \Delta \omega}{3(4+p)^{2} C_{2}^{2}} \sinh ^{2}\left(\sqrt{\frac{3(4+p)^{2}}{128}} C_{2} \tau\right)
$$

Conseqüentemente,

$$
\begin{aligned}
e^{\alpha} & =\left(\frac{8 \Delta \omega}{3(4+p)^{2} C_{2}^{2}}\right)^{\frac{2 p}{\Delta}} e^{\frac{(4+p)(2-p)}{4 \Delta}\left(C_{1}+C_{2} \tau\right)} \sinh ^{\frac{2 p}{\Delta}}\left(\sqrt{\frac{3(4+p)^{2}}{128}}\left|C_{2} \tau\right|\right) \\
e^{\beta} & =\left(\frac{3(4+p)^{2} C_{2}^{2}}{8 \Delta \omega}\right)^{\frac{4+p}{\Delta}} \frac{\exp \left(-\frac{3(4+p)}{2 \Delta}\left(C_{1}+C_{2} \tau\right)\right)}{\sinh ^{2 \frac{4+p}{\Delta}}\left(\sqrt{\frac{3(4+p)^{2}}{128}} \mid C_{2} \tau\right) \mid}
\end{aligned}
$$

No limite $\tau \rightarrow 0$ (3.91) se reduz a (3.77), portanto a relação $\tau=f(t)$ tende à forma (3.78). Então, os fatores de escala terão o comportamento assintótico dado por (3.81), (3.82) e a relação entropia/área será descrita por (3.84) no futuro.

Para o caso $\left(\Delta<0(p>0.899), C_{2}<0\right)$ a solução pode ser encontrada com a substituição

$$
e^{-X}=\frac{8|\Delta| \omega}{3(4+p)^{2} C_{2}^{2}} \cosh ^{2}(\theta)
$$

A equação para $\theta$ é a mesma que no caso anterior e a solução é

$$
e^{-X}=\frac{8|\Delta| \omega}{3(4+p)^{2} C_{2}^{2}} \cosh ^{2}\left(\sqrt{\frac{3(4+p)^{2}}{128}} C_{2} \tau\right) .
$$

Então, os fatores de escala ficam

$$
\begin{aligned}
e^{\alpha} & =\left(\frac{8|\Delta| \omega}{3(4+p)^{2} C_{2}^{2}}\right)^{\frac{2 p}{|\Delta|}} e^{\frac{(4+p)(\Delta-p)}{4|\Delta|}\left(C_{1}+C_{2} \tau\right)} \cosh ^{\frac{2 p}{|\Delta|}}\left(\sqrt{\frac{3(4+p)^{2}}{128}}\left|C_{2} \tau\right|\right) \\
e^{\beta} & =\left(\frac{8|\Delta| \omega}{3(4+p)^{2} C_{2}^{2}}\right)^{-\frac{4+p}{|\Delta|}} \frac{\exp \left(-\frac{3(4+p)}{2|\Delta|}\left(C_{1}+C_{2} \tau\right)\right)}{\cosh ^{2 \frac{4+p}{\Delta \mid}}\left(\sqrt{\frac{3(4+p)^{2}}{128}}\left|C_{2} \tau\right|\right)}
\end{aligned}
$$


Esta solução toma a forma tipo Kasner de modo assintótico (ver [68]). Em outras palavras, os fatores de escala $a(t)$ e $b(t)$ tomam a forma (3.61),(3.62) no futuro. Isto implica que a razão entropia/área coincidirá com aquela do caso $\Delta=0$ no futuro remoto (ver figura 3.4).

Portanto, o princípio holográfico é satisfeito na época de contração do modelo apresentado. A razão $S / A$ tem um comportamento assintótico similar para todas as soluções. 


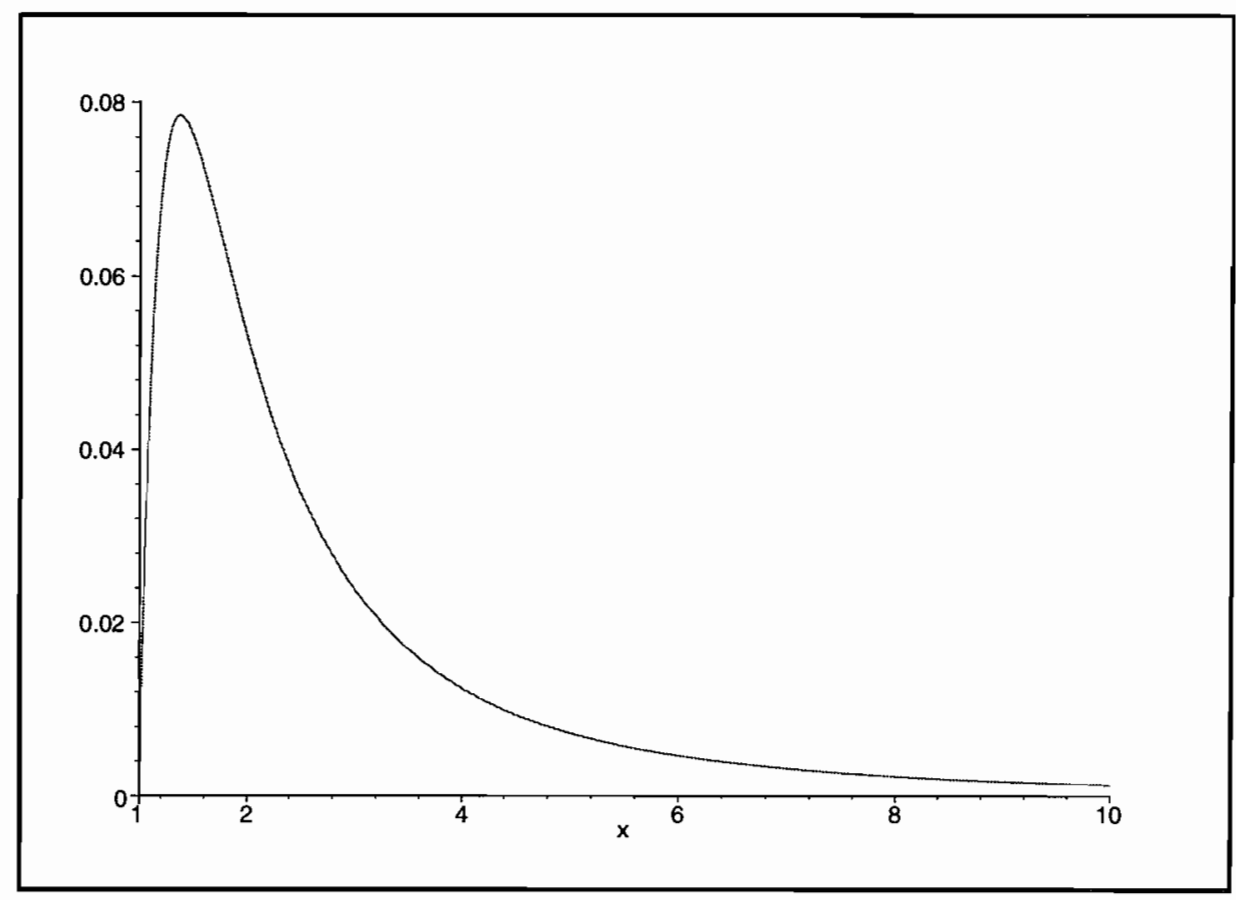

Figura 3.1: Função $f(x)$ no caso crítico $p=0.899$. 


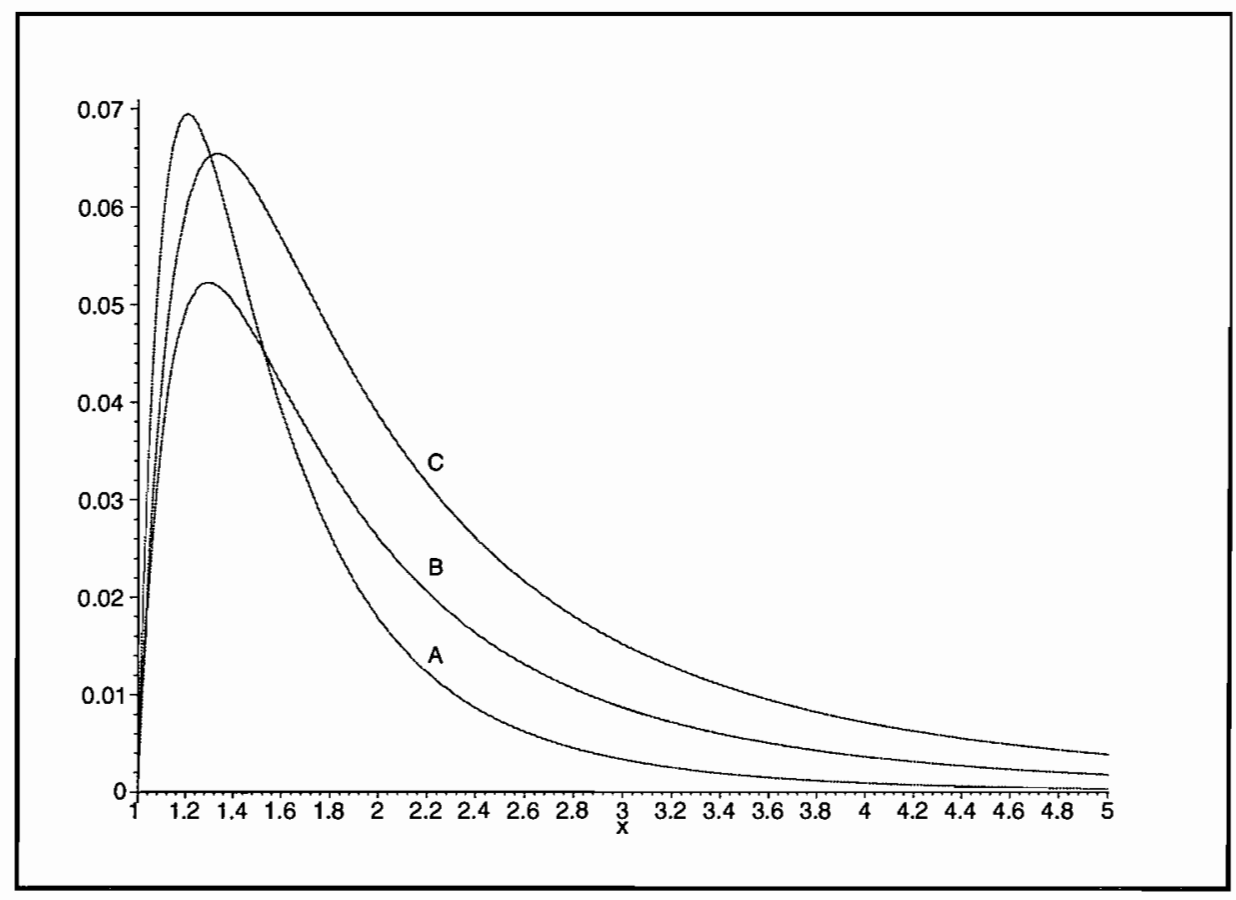

Figura 3.2: Função $g(x)$ nos casos subcríticos $p=0(A), 0.5(B), 0.7(C)$. 


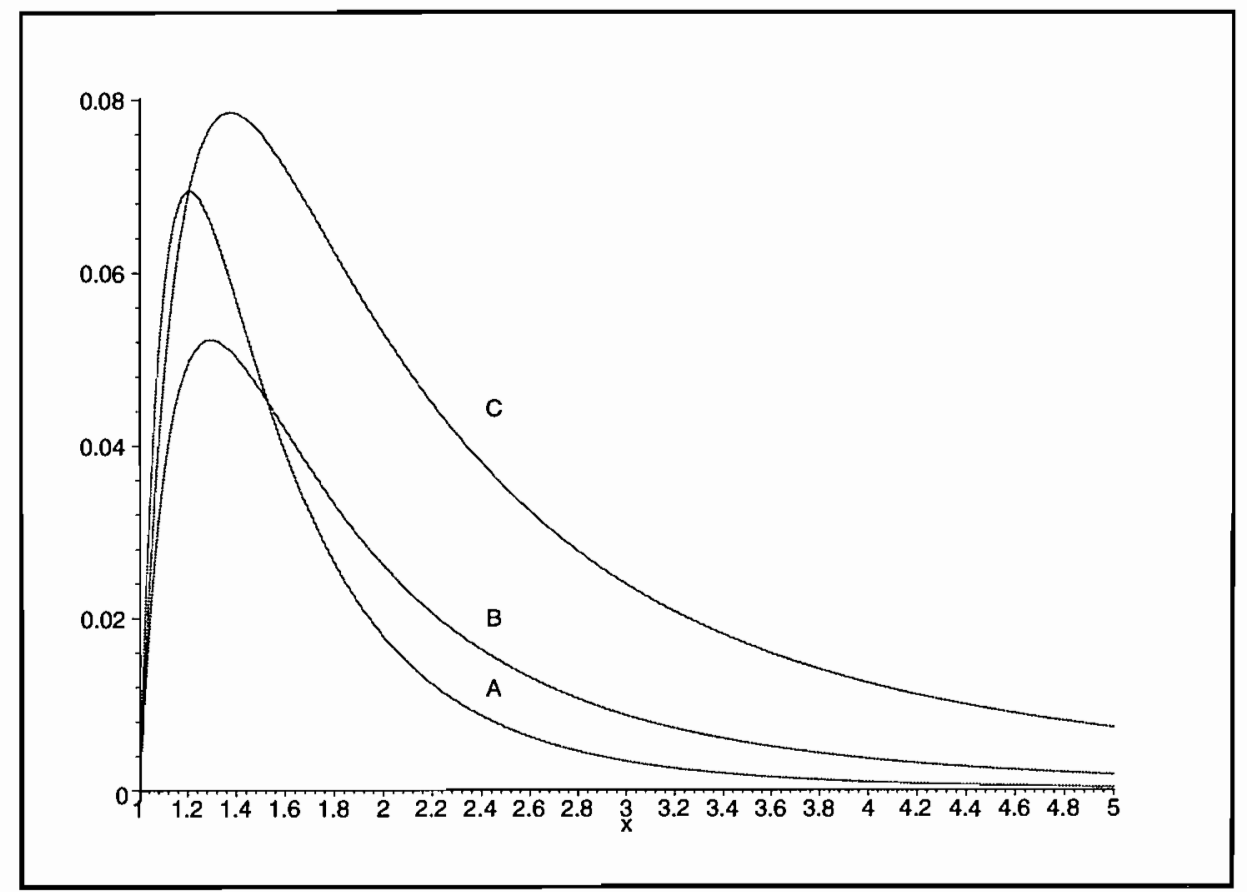

Figura 3.3: Comparação entre os casos $p=0(A), 0.5(B), 0.899(C)$. 


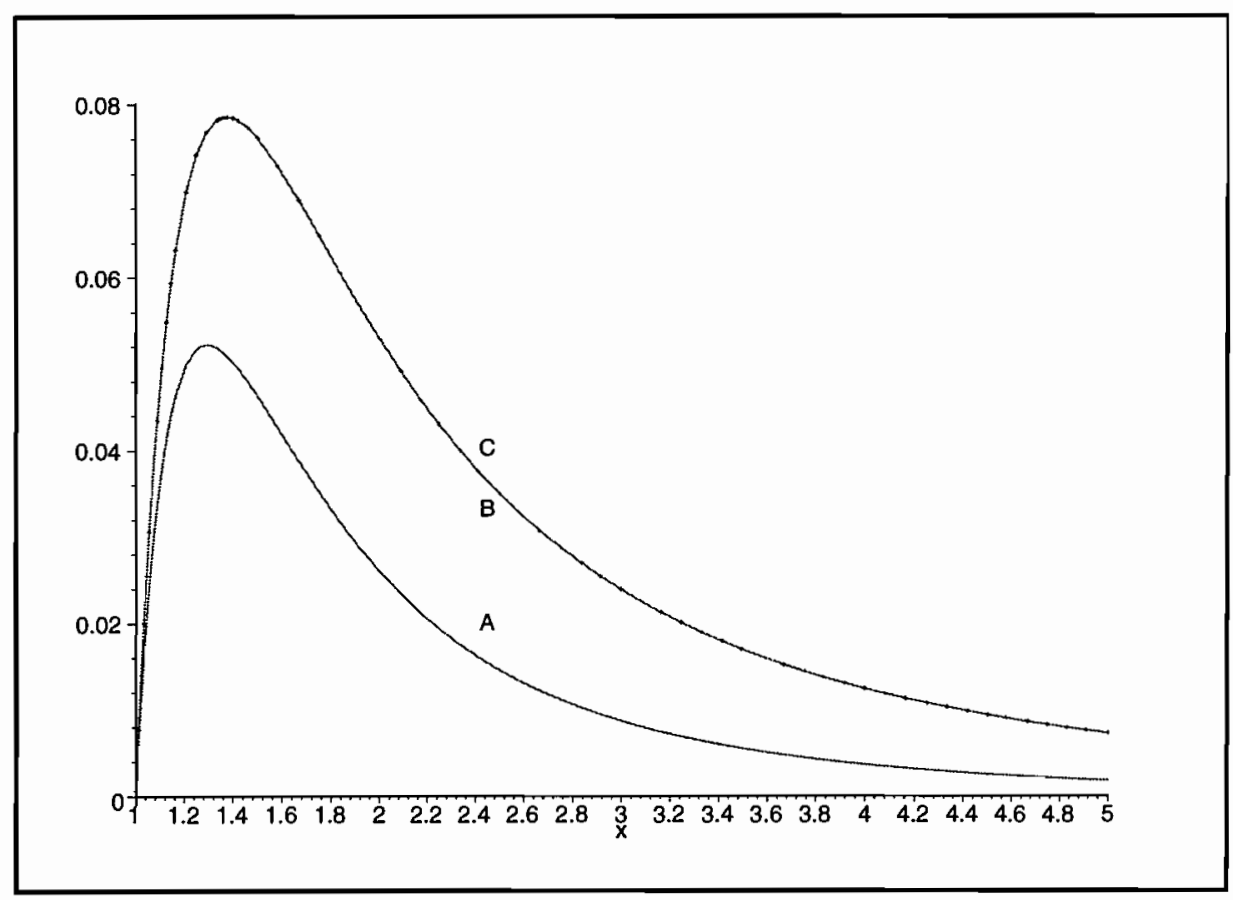

Figura 3.4: Comparação entre os casos $p=0.5(A), 0.899(B), 8(C)$. 


\subsection{Fórmula de Cardy-Verlinde}

Para finalizar este capítulo gostariamos de apresentar uma idéia recente proposta por E. Verlinde [77] que pode ajudar significativamente na compreensão da conexão entre holografia e cosmologia.

O Princípio Holográfico em sua forma mais simples, $S<A / 4 G$, não pode ser aplicado para um universo fechado porque o espaço não tem fronteiras. Portanto precisa-se de uma versão modificada da holografia.

A radiação num universo tipo FRW é usualmente descrita por partículas sem massa que são livres ou interagem fracamente. De modo mais geral, a radiação pode ser descrita por uma teoria de campos conforme (TCC) com interação. O número de espécies de partículas sem massa é refletido no valor da carga central $c$ da TCC. Aqui a radiação é caracterizada por uma TCC com uma carga central muito grande. Num volume finito a energia $E$ tem uma contribuição tipo Casimir proporcional a $c$. Devido a este efeito Casimir a entropia não é mais uma função extensiva de $V$ e $E$. A entropia de uma TCC em duas dimensões é dada pela conhecida fórmula de Cardy [44]

$$
S=2 \pi \sqrt{\frac{c}{6}\left(L_{0}-\frac{c}{24}\right)}
$$

onde $L_{0}$ representa o produto $E R$ da energia e o raio, e a subtração $\frac{c}{24}$ é causada pelo efeito Casimir. Mostraremos, que depois de fazer as identificações apropriadas para $L_{0}$ e $c$, a mesma fórmula de Cardy é válida também para teorias de campo conformes em outras dimensões. Este resultado é surpreendente porque a obtenção desta fórmula é baseada na invariança modular que só acontece em duas dimensões.

Agora mostramos como Verlinde chegou a este resultado. Em $n+1$ dimensões as equações de FRW são dadas por

$$
\begin{aligned}
H^{2} & =\frac{16 \pi G}{n(n-1)} \frac{E}{V}-\frac{1}{R^{2}} \\
\dot{H} & =-\frac{8 \pi G}{n-1}\left(\frac{E}{V}+p\right)+\frac{1}{R^{2}}
\end{aligned}
$$

onde $H=\dot{R} / R$ é o parâmetro de Hubble. As equações de FRW usualmente são escritas em termos da densidade de energia $\rho=E / V$, mas no presente caso é mais conveniente trabalhar com a energia total. 
O surpreendente acontece quando as substituições

$$
\begin{array}{rll}
2 \pi L_{0} & \Rightarrow & \frac{2 \pi}{n} E R \\
2 \pi \frac{c}{12} & \Rightarrow & (n-1) \frac{V}{4 G R} \\
S & \Rightarrow & (n-1) \frac{H V}{4 G}
\end{array}
$$

são introduzidas na equação de Cardy e obtemos a equação de Friedmann (3.96) em $n+1$ dimenções. Então podemos dizer que de algum modo a equação de Friedmann conhece sobre a fórmula de Cardy. Isto pode indicar que as duas equações emergem de alguma teoria fundamental subjacente.

Obviamente este resultado não é uma prova. Portanto muitos trabalhos foram feitos tentando conferir a validade da chamada fórmula de CardyVerlinde que é a fórmula de Cardy em dimensões maiores que dois.

Este resultado foi extendido para $\Lambda \neq 0$ no trabalho [78]. 


\section{Capítulo 4}

\section{Conclusões}

Neste trabalho estudamos a entropia e testamos seu chamado comportamento holográfico na gravidade. No caso dos buracos negros o método de 't Hooft foi aplicado em diversas situações. Para buracos negros com cargas em $D=$ 4,5 obtivemos que a entropia é proporcional à área, em primeira ordem, como era esperado. A presença de um fator extra nos elemento de linha, devido as cargas, não modifica a dependência da entropia com respeito à área. Mas quando foi testado o método para os buracos da gravidade de Lovelock o resultado mostrou as limitações deste procedimento. Também neste foi encontrada a proporcionalidade da entropia com á área, independentemente dos parâmetros $D$ e $k$. Mas a entropia, obtida da termodinâmica, para este tipo de buraco negro, depende de $r_{h}^{D-2 k}$. Portanto os resultados não coincidem e conseqüentemente as divergências não podem ser absorvidas pela constante de Newton. Então podemos concluir que o método da parede de tijolos é "linear" no sentido que só reproduz a entropia de buracos negros na gravidade de Einstein-Hilbert. Uma possível explicação para este fato pode ser que o procedimento de 't Hooft calcula, por construção, os modos que moram numa camada esférica e no final dos cálculos isto sempre fica refletido.

Continuando com a gravidade de Lovelock aplicamos o limite de Bekenstein da entropia para os buracos negros desta teoria. O resultado foi muito parecido com o caso do buraco negro de Schwarzschild, mas com um fator extra dependente da dimensão e da potência da curvatura, $k$. No caso de um corpo (autogravidade fraca) jogado neste tipo de buraco negro, o resultado foi o esperado, e não depende das características do buraco negro.

Passando à cosmologia, as soluções do modelo cosmológico de ArkaniHamed et al no período de contração foram estudadas. Usando o critério 
de Fischler e Susskind(FS) para universos anisotrópicos a grandeza $S / A$ foi calculada para cada uma delas. Em todos os casos foi conferido que $S / A<1$ e portanto o modelo é "holográfico". Um elemento positivo deste cálculo foi a extensão do critério FS para inflação, mas sem o processo de "reaquecimento" padrão.

O estudado nesta tese nos motiva a aprofundar em duas questões apresentadas. Primeiramente seria muito útil comprender quais são os pontos de contacto das diferentes abordagens para se obter os graus de liberdade do buraco negro. Uma verdadeira comprensão deste tópico nos levaria a uma melhoria significativa no caminho da unificação entre a Relatividade Geral e a Mecânica Quântica. A outra questão a estudar no futuro seria a procura por critérios holográficos mais adequados na cosmologia. Até agora existem muitas opiniões diferentes na literatura sobre a aplicação da holografia na cosmologia. 


\section{Apêndice A}

\section{Entropia de Gibbons-Hawking}

A ação para o campo gravitacional é

$$
\frac{1}{16 \pi G} \int R(-g)^{1 / 2} d^{4} x
$$

A curvatura escalar $R$ contém termos que são lineares na segunda derivada da métrica. Com o objetivo de se obter uma ação que dependa unicamente das primeiras derivadas da métrica, como é exigido pelas integrais de caminho, as segundas derivadas têm que ser removidas com a integração por partes. Portanto a ação para a métrica $g$ na região $Y$ com fronteira $\partial Y$ tem a forma [56]

$$
I=\frac{1}{16 \pi G} \int_{Y} R(-g)^{1 / 2} d^{4} x+\frac{1}{8 \pi G} \int_{\partial Y}[K](-h)^{1 / 2} d x^{3},
$$

onde $K$ denota a curvatura extrínseca e $[K]$ representa a diferença desta grandeza para a métrica do buraco negro com métrica do espaço chato de Minkowski, ou seja

$$
[K]=K^{B N}-K^{c h} .
$$

A função de partição na aproximação do ponto de sela é

$$
Z \approx e^{-I} \text {. }
$$

A aproximação semiclássica é implementada substituindo na ação uma solução clássica, neste caso a métrica Euclidiana de Schwarzschild,

$$
d s^{2}=\left(1-\frac{2 M G}{r}\right) d \tau^{2}+\left(1-\frac{2 M G}{r}\right)^{-1} d r^{2}+r^{2} d \Omega^{2} .
$$


Portanto $R=0$, e o valor da ação é completamente determinado pelo termo de superfície. Com este objetivo usamos a relação

$$
\int(-h)^{1 / 2} K d \sigma=\frac{\partial}{\partial n} \int(-h)^{1 / 2} d \sigma
$$

onde $(\partial / \partial n) \int d \sigma$ é a derivada da área $\int^{*} d \sigma$ de $\partial Y$. Deste modo,

$$
\begin{aligned}
\int(-h)^{1 / 2} K^{B N} d \sigma & =-\left(1-\frac{2 M G}{r}\right)^{1 / 2} \frac{\partial}{\partial r}\left[4 \pi r^{2}\left(1-\frac{2 M G}{r}\right)^{1 / 2} \beta\right] \\
& =-8 \pi r \beta+12 \pi G M \beta
\end{aligned}
$$

Para a métrica do espaço chato temos

$$
\int(-h)^{1 / 2} K^{C H} d \sigma=-8 \pi r \beta+8 \pi G M \beta
$$

e obtemos

$$
\ln Z=-\frac{1}{8 \pi G \hbar} \int d \sigma(-h)^{1 / 2}[K]=-\frac{\beta^{2}}{16 \pi G \hbar} .
$$

Agora, a partir da fórmula termodinâmica padrão

$$
S=-\beta^{2} \frac{\partial}{\partial \beta}\left(\frac{1}{\beta} \ln Z\right)
$$

obtemos

$$
S=\hbar \frac{\beta^{2}}{16 \pi G}
$$

Usando a relação entre $\beta$ e $M$ chegamos ao resultado

$$
S=\frac{4 \pi G M^{2}}{\hbar}=\frac{A}{4 G \hbar}
$$

que é a conhecida fórmula de Bekenstein-Hawking. 


\section{Apêndice B}

\section{Formalismo Hamiltoniano para a ação de Lovelock.}

A forma Hamiltoniana de (2.65) é discutida em [31]. Como em $D=4$, as coordenadas canônicas são as componentes espaciais da métrica $g_{i j}$, e seu momentum conjugado é $\pi^{i j}$. As componentes temporais $g_{0 \mu}$ são os multiplicadores de Lagrange associados com os geradores das deformações de superficie, $\mathcal{H}_{\mu}=\left(\mathcal{H}, \mathcal{H}_{i}\right)$. Então, neste contexto, a ação (2.65) toma a forma

$$
I=\int\left(\pi^{i j} \dot{g}_{i j}-N \mathcal{H}-N^{i} \mathcal{H}_{i}\right) d^{D-1} x d t+B
$$

Os momentos ficam sendo

$$
\pi_{j}^{i}=-\frac{1}{4} \sqrt{g} \sum \frac{\alpha_{p}}{2 p !} \frac{(D-2 p) !}{2^{p}} \sum_{s=0}^{p-1} C_{s(p)} \delta_{\left[j_{i} \ldots j_{2 p-1}\right]}^{\left[i_{1} \ldots i_{2 p-1} i\right]} R_{i_{1} i_{2}}^{j_{1} j_{2}} \ldots R_{i_{2 s-1} i_{2 s}}^{j_{2 p-1} j_{2 s}} K_{i_{2 s+1}}^{j_{2 s+1}} \ldots K_{i_{2 p-1}}^{j_{2 p-1}}
$$

onde

$$
C_{s(p)}=\frac{(-4)^{p-s}}{s ![2(p-s)-1] ! !} .
$$

Os geradores das reparametrizações nas superfícies $t=$ const são

$$
\mathcal{H}_{i}=-2 \pi_{i / l}^{l},
$$

onde a barra oblíqua denota a diferenciação covariante na métrica espacial, isto é $\pi_{j / i}^{i}=\pi_{j, i}^{i}-\Gamma_{j i}^{n} \pi_{n}^{i}$. 
O gerador normal $\mathcal{H}$ é

$$
\mathcal{H}=-\sqrt{\operatorname{det}\left(g_{i j}\right)} \sum_{p=0}^{n-1} \frac{(D-2 p) !}{2^{p}} \alpha_{p} \tilde{R}_{i_{1} i_{2}}^{j_{1} j_{2}} \tilde{R}_{i_{3} i_{4}}^{j_{3} j_{4}} . \tilde{R}_{i_{2 p-1} i_{2 p}}^{j_{2 p-1} j_{2 p}}
$$

onde $\tilde{R}_{k l}^{i j}$ são as componentes do tensor de curvatura e dependem das velocidades através das equações de Gauss-Codazzi

$$
\tilde{R}_{i j k l}=R_{i j k l}+K_{i k} K_{j l}-K_{i l} K_{j k}
$$

onde $K_{i j}=\frac{1}{2 N}\left(-\dot{g}_{i j}+N_{i / j}+N_{j / i}\right)$.

No caso estático e esfericamente simétrico não há distinção entre $\tilde{R}_{i j k l} \mathrm{e}$ $R_{i j k l}$. Também, as únicas componentes que não se anulam são

$$
\begin{aligned}
R_{n_{1} n_{2}}^{m_{1} m_{2}} & =\frac{h(r)}{r^{2}} \delta_{\left[n_{1} n_{2}\right]}^{\left[m_{1} m_{2}\right]} \\
R_{r n}^{r m} & =\frac{h^{\prime}(r)}{2 r} \delta_{n}^{m}
\end{aligned}
$$

onde $h(r)=1-g^{r r}(r)$.

$O$ gerador normal $\mathcal{H}$ pode ser calculado por uma substituição direta de (B.7) em (B.5) obtendo-se

$$
\mathcal{H}=-(D-2) ! \sqrt{\gamma} g^{-1} \frac{d}{d r}\left[r^{d-1} \sum \alpha_{p}(D-2 p)\left(\frac{1-f^{2}}{r^{2}}\right)^{p}\right]
$$

Colocando-se nesta última expressão a escolha(2.72) para os coeficientes $\alpha_{p}$ e levando-se em conta que satisfazem a relação

$$
\sum_{p}^{\frac{D-1}{2}}(D-2 p) \alpha_{p}\left(\frac{1-f^{2}}{r^{2}}\right)^{p}=\prod_{i}^{k}\left(\frac{1-f^{2}}{r^{2}}+\frac{1}{\ell^{2}}\right)
$$

a ação (2.79) é obtida. 


\section{Apêndice C}

\section{Constantes}

Aqui mostramos os valores calculados [38] das constantes $C_{(d)}^{(k)}$ na expressão de energia livre (2.119) para diversos valores da dimensão $d$ e o grau na curvatura $k$ :

$$
\begin{aligned}
C_{(6)}^{(2)} & =\frac{64 \zeta(6)}{3 \pi} \\
C_{(7)}^{(2)} & =\frac{3 \zeta(7)}{4} \\
C_{(8)}^{(3)} & =\frac{243^{3} \zeta(8)}{5 \pi} \\
C_{(9)}^{(2)}=\frac{5 \zeta(9)}{2^{9 / 2} 16} & C_{(9)}^{(3)}=\frac{5 \zeta(9)}{16} \\
C_{(10)}^{(2)}=\frac{2^{11} \zeta(10)}{7 \pi 5^{6}} \quad C_{(10)}^{(3)} & =\frac{8^{2} \zeta(10)}{35 \pi} \quad C_{(10)}^{(4)}=\frac{2^{16} \zeta(10)}{35 \pi} .
\end{aligned}
$$




\section{Apêndice D}

\section{Supercordas e D-branas}

\section{D.1 Supercordas}

A ação de supercordas NS-R é

$$
S=\int_{\mathcal{M}} d^{2} \sigma \frac{1}{2 \pi}\left(\partial_{a} X_{\mu} \partial^{a} X^{\mu}-i \bar{\psi}^{\mu} \rho^{a} \partial_{a} \psi_{\mu}\right)
$$

onde $a=1,2$ e $\mu$ é um indice de espaço-tempo.

Note-se também que $\psi$ é um objeto sui generis, um spinor de Majorana em duas dimensões e um vetor no espaço-tempo. Definamos

$$
\begin{gathered}
\psi^{\mu}=\left(\begin{array}{c}
\psi_{0}^{\mu} \\
\psi_{1}^{\mu}
\end{array}\right) \\
\rho^{0}=\left(\begin{array}{cc}
0 & -i \\
i & 0
\end{array}\right) \\
\rho^{1}=\left(\begin{array}{cc}
0 & i \\
i & 0
\end{array}\right) \\
\left\{\rho^{a}, \rho^{b}\right\}=-2 \eta^{a b}
\end{gathered}
$$

Contrariamente à corda bosônica, aqui temos duas condições de fronteira diferentes que podemos colocar para os campos $\psi_{0}$ e $\psi_{1}$. No ponto $\sigma=0$ sempre podemos escolher $\psi_{0}=\psi_{1}$. Contudo, no ponto $\sigma=\pi$ podemos 
escolher duas condições de fronteira diferentes. Se o campo e periódico (antiperiódico), temos a condição de fronteira tipo Ramond (Neveu-Schwarz):

$$
\begin{cases}\text { Ramond } & \psi_{0}(\pi, \tau)=\psi_{1}(\pi, \tau) \\ \text { Neveu-Schwarz } & \psi_{0}(\pi, \tau)=-\psi_{1}(\pi, \tau)\end{cases}
$$

Como temos dois condições de fronteira diferentes para o spinor é natural ter também dois modos para expandir esses campos em componentes de Fourier:

$$
\begin{gathered}
R:\left\{\begin{array}{l}
\psi_{0}^{\mu}(\sigma, \tau)=2^{-1 / 2} \sum_{-\infty}^{\infty} d_{n}^{\mu} e^{-i n(\tau-\sigma)} \\
\psi_{1}^{\mu}(\sigma, \tau)=2^{-1 / 2} \sum_{-\infty}^{\infty} d_{n}^{\mu} e^{-i n(\tau+\sigma)} .
\end{array}\right. \\
N S:\left\{\begin{array}{l}
\psi_{0}^{\mu}(\sigma, \tau)=2^{-1 / 2} \sum_{r \in \mathbf{Z}+1 / 2} b_{r}^{\mu} e^{-i r(\tau-\sigma)} \\
\psi_{1}^{\mu}(\sigma, \tau)=2^{-1 / 2} \sum_{r \in \mathbf{Z}+1 / 2} b_{r}^{\mu} e^{-i r(\tau+\sigma)} .
\end{array}\right.
\end{gathered}
$$

A Hamiltoniana no setor NS é

$$
H=\sum_{n=1}^{\infty} n a_{-n}^{\mu} a_{n \mu}+\sum_{r=1 / 2}^{\infty} r b_{-r}^{\mu} b_{r \mu}+\alpha^{\prime} p_{0}^{2}
$$

No setor $\mathrm{R}$ é

$$
H=\sum_{n=1}^{\infty} n a_{-n}^{\mu} a_{n \mu}+\sum_{m=1}^{\infty} m d_{-m}^{\mu} d_{m \mu}+\alpha^{\prime} p_{0}^{2}
$$

O espaço de Fock fica

$$
\begin{aligned}
& N S: \quad \prod_{n, \mu} \prod_{m, \nu}\left\{a_{n, \mu}^{\dagger}\right\}\left\{b_{m, \nu}^{\dagger}\right\} \mid 0> \\
& R: \quad \prod_{n, \mu} \prod_{m, \nu}\left\{a_{n, \mu}^{\dagger}\right\}\left\{d_{m, \nu}^{\dagger}\right\} \mid 0>u_{\alpha}
\end{aligned}
$$

onde $u_{\alpha}$ é um spinor arbitrário.

$\mathrm{Na}$ teoria de supercordas tipo II as cordas são fechadas e orientadas. Para os fermions podemos escolher as condições de fronteira independentemente para os modos que se movem à esquerda daqueles que se movem à direita. Portanto temos quatro tipos de condições de fronteiras conhecidas como NSNS, NS-R, R-NS e R-R. Como o vacuo do setor R leva uma representação da álgebra de Clifford então é um espinor no espaço tempo. Portanto os estados dos setores NS-NS e R-R são bosônicos enquanto os estados nos setores NS-R e R-NS são fermiônicos. 


\section{D.2 D-branas}

A ação da corda bosônica é (no gauge conforme)

$$
\frac{1}{4 \pi \alpha^{\prime}} \int_{M} d \sigma d \tau \partial_{a} X^{\mu} \partial_{a} X_{\mu}
$$

Variando com respeito a $X^{\mu}$ e integrando por partes,

$$
\delta S=-\frac{1}{2 \pi \alpha^{\prime}} \int_{M} d \sigma d \tau \delta X^{\mu} \partial^{2} X_{\mu}+\frac{1}{2 \pi \alpha^{\prime}} \int_{\delta M} d \tau \delta X^{\mu} \partial_{\sigma} X_{\mu}
$$

onde $\partial_{\sigma}$ é a derivada normal à fronteira. A condição de ação mínima nos leva à equação de Laplace $\partial^{2} X_{\mu}=0$. Do termo de fronteira obtemos a condição

$$
\left.\delta X^{\mu} \partial_{\sigma} X_{\mu}\right|_{0, \pi}=0,
$$

separadamente nos extremos da corda aberta $\sigma_{1}=0$ e $\sigma_{1}=\pi$. Portanto duas condições de fronteira podem ser satisfeitas: a condição de Neumann

$$
\partial_{n} X^{\mu}=0
$$

ou a condicão de Dirichlet

$$
\delta X^{\mu}=0
$$

A solução geral da equação de Laplace com condições de fronteira de Newmann é

$$
X^{\mu}(z, \bar{z})=x^{\mu}-i \alpha^{\prime} p^{\mu} \ln (z \bar{z})+i \sqrt{\frac{\alpha^{\prime}}{2}} \sum \frac{\alpha_{m}^{\mu}}{m}\left(z^{-m}+\bar{z}^{-m}\right)
$$

onde $x_{\mu}$ e $p^{\mu}$ são a posição e momentum do centro de massa. Como é usual numa teoria conforme escrevemos em termos da coordenada $z=e^{\sigma^{2}-i \sigma^{1}}$.

Os comutadores da quantização canônica são

$$
\begin{aligned}
{\left[x^{\mu}, p^{\nu}\right] } & =i \eta^{\mu \nu} \\
{\left[\alpha_{m}^{\mu}, \alpha_{n}^{\nu}\right] } & =m \delta_{m+n} \eta^{\mu \nu} .
\end{aligned}
$$

A condição de massa é

$$
M^{2}=-p^{\mu} p_{\mu}=\frac{1}{\alpha^{\prime}}\left(\sum_{m=1}^{\infty} m N_{m}-1\right),
$$


onde $N_{m}$ é o número de osciladores excitados no modo $m$, e o -1 é a energia de ponto zero dos bósons.

Agora, podemos mostrar o conceito de "D-branas". São definidas como superfícies p-dimensionais extendidas no espaço-tempo e onde as cordas podem terminar, isto é

$$
\begin{aligned}
\partial_{\sigma} X^{\mu}=0 & \text { para } \mu=0, \ldots, p \quad N, \\
X^{\mu}=0 & \text { para } \mu=p+1, \ldots, 9 \quad D,
\end{aligned}
$$

que representa uma Dp-brana localizada em $X_{p+1}=\ldots . . X_{9}=0$.

No caso de se ter duas branas a situação é mais geral porque os extremos da corda podem estar em branas distintas. No caso em que sejam duas Dp branas com dimensões diferentes (conhecido como sistema $p-p^{\prime}$ ) teremos quatro tipos de condições de fronteira.

Se ambos os extremos satisfazem á condição de Neumann (NN) obtemos

$$
X^{\mu}(z, \bar{z})=x^{\mu}-i \alpha^{\prime} p^{\mu} \ln (z \bar{z})+i \sqrt{\frac{\alpha^{\prime}}{2}} \sum_{m \ni 0} \frac{\alpha_{m}^{\mu}}{m}\left(z^{-m}+\bar{z}^{-m}\right) .
$$

No caso de condições de fronteira tipo DD temos

$$
X^{\mu}(z, \bar{z})=i \frac{\delta X^{\mu}}{2 \pi} \ln (z / \bar{z})+i \sqrt{\frac{\alpha^{\prime}}{2}} \sum_{m \ni 0} \frac{\alpha_{m}^{\mu}}{m}\left(z^{-m}+\bar{z}^{-m}\right)
$$

Finalmente, no caso de condições de froteira mistas $\mathrm{DN}(+), \mathrm{ND}(-)$ temos

$$
X^{\mu}(z, \bar{z})=i \sqrt{\frac{\alpha^{\prime}}{2}} \sum_{r \in Z+1 / 2} \frac{\alpha_{r}^{\mu}}{r}\left(z^{-r} \pm \bar{z}^{-r}\right)
$$

A ação de supergravidade para baixas energias contem $p+1$ formas $A_{p+1}$ que vem do sector RR, $p$ é par no caso IIA e ímpar no caso IIB. Não existem objetos na teoria de cordas (perturbativa) que portem a carga desses campos. Os objetos que portariam carga de uma $p+1$ forma são as p-branas. $\mathrm{O}$ acoplamento é

$$
\mu_{p} \int_{V_{p+1}} A_{p+1}
$$

isto é uma generalização do acoplamento eletromagnético a uma carga elétrica. De fato, as teorias de supergravidade tipo II contém soluções de $p$-branas que portam carga. 


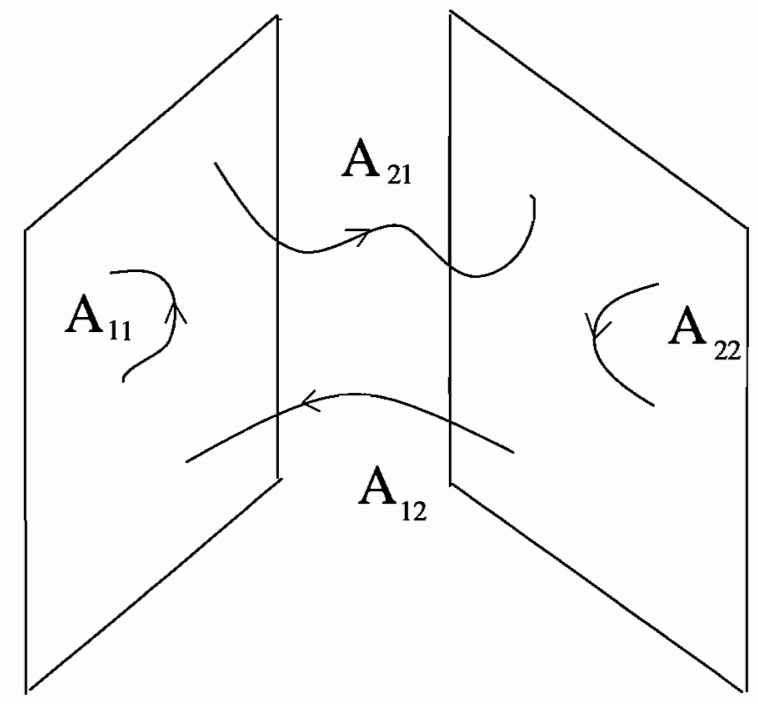

Figura D.1: Um sanduiche de duas D-branas com quatro cordas abertas. 


\section{Referências Bibliográficas}

[1] G.t'Hooft, Dimensional Reduction in Quantum Gravity, gr-qc/9310026.

[2] L. Susskind J. Math. Phys 36 (1995) 63

[3] J.D. Bekenstein, Phys. Rev. D7 (1973), 949.

[4] S. Hawking, Commun. Math. Phys. 43 (1975) 199.

[5] J. Maldacena, Adv. Theor. Math. Phys. 2 (1998) 231.

[6] E. Witten, Adv. Theor. Math. Phys. 2 (1998) 253.

[7] S.S. Gubser, I.R. Klebanov and A.M. Polyakov, Phys. Lett. B428 (1998) 105.

[8] O. Aharony, S.S. Gubser, J. Maldacena and H. Ooguri, Y. Oz, Phys. Rept. 323 (2000) 183.

[9] S. Carlip, Nucl. Phys. Proc. Suppl. 88 (2000) 10.

[10] A. Strominger, JHEP 9802 (1998) 009.

[11] A. Ashtekar, J. Baez, A. Corichi and K. Krasnov, Phys. Rev. Lett. 80 (1998) 904.

[12] C. Rovelli, Strings, loops and others: a critical survey of the present approaches to quantum gravity, gr-qc/9803024.

[13] G.t'Hooft, Nucl. Phys B256(1985) 727.

[14] A.J.M. Medved, Brick Walls on the Brane, hep-th/0110118.

[15] E. Winstanley, Phys. Rev. D63 (2001) 084013. 
[16] N.E. Mavromatos and E. Winstanley, Phys. Rev. D53 (1996) 3190.

[17] F. Larsen and F. Wilczek, Nucl. Phys. B458 (1996) 249.

[18] E. Abdalla and L.A. Correa-Borbonet, Mod. Phys. Lett. A16 (2001) 2495 .

[19] K. Shiraishi, Mod. Phys. Lett. A9 (1994) 3509.

[20] J.G. Demers, R. Lafrance and R.C. Myers, Phys. Rev. D52 (1995) 2245.

[21] N.D. Birrell and P.C. Davies, Quantum Field Theory in Curve Space(Cambridge University Press, Cambridge 1982); S.M. Christensen, Phys. Rev. D14 (1976) 2490.

[22] T. Mohaupt, hep-th/0004098

[23] S. Mukohyama and W. Israel, Phys. Rev. D58, (1998) 104005.

[24] L. Susskind and J. Uglum, Phys. Rev. D50 (1994) 2700.

[25] D. Lovelock, J. Math. Phys. 12 (1971) 498.

[26] J.Z. Simon, Phys. Rev. D41(1990) 3720.

[27] F. Muller-Hoissen, Nucl. Phys. B337 (1990) 709.

[28] N. Deruelle and L. Farina-Busto, Phys. Rev. D41(1990) 3696.

[29] D. Lovelock and H. Rund, Tensors differential forms, and variational principles.

[30] J. Crisostomo, R. Troncoso and J. Zanelli, Phys. Rev. D62(2000) 084013.

[31] M. Bañados, C. Teitelboim and J. Zanelli, Phys. Rev.D49, (1994) 975.

[32] J.T. Wheeler, Nucl. Phys. B268 (1986) 737.

[33] F. Muller-Hoissen, Phys. Lett B163 (1985) 106.

[34] B. Zumino, Phys. Rep 137 (1986) 109. 
[35] J. Demaret, H. Caprasse, A. Moussiaux, Ph. Tombal and D. Papadopoulos, Phys. Rev D41 (1990) 1163.

[36] B. Whitt, Phys. Rev. D38 (1988) 3001.

[37] R.C. Myers and J. Simon, Phys. Rev.D38 (1988) 2434.

[38] E. Abdalla and L.A. Correa-Borbonet, Aspects of Higher Order Gravity and Holography, hep-th/0109129.

[39] Higher Transcendental Functions(Bateman Manuscript Proyect), edited by A. Erdelyi et al(McGraw-Hill, New York, 1953).

[40] A.A. Belavin, A.M. Polyakov and A.B. Zamolodchikov, Nucl. Phys. B241 (1984) 333 .

[41] S.V. Ketov, Conformal field theory. Singapore: World Scientific, (1995).

[42] M. Kaku, Strings, Conformal Fields and Topology: An Introduction. New York, USA: Springer (1991) 535 p. (Graduate texts in contemporary physics).

[43] M. A. Virasoro, Phys. Rev. D1 (1970) 2933.

[44] J.L. Cardy, Nucl. Phys. B270 (1986) 186.

[45] Sergey N. Solodukhin, Phys. Lett. B454 (1999) 213.

[46] S. Carlip, Class. Quant. Grav. 15, (1998) 3609.

[47] M. Green, J. Schwarz and E. Witten, "Superstring Theory", Vol 1 2, Cambridge University Press, (1987).

[48] J. Polchinski, Phys. Rev. Lett. 75 (1995) 4724.

[49] J. Polchinski, hep-th/9611050.

[50] E.Witten, Nucl. Phys. B460 (1996) 335.

[51] A. Strominger and C. Vafa, Phys. Lett. B379 (1996) 99.

[52] C.G. Callan, J.M. Maldacena, Nucl. Phys. B472 (1996) 591.

[53] J.M. Maldacena Black Holes in String Theory hep-th/9607235. 
[54] S.F. Hassan, S.R. Wadia, Phys. Lett. B402 (1997) 43.

[55] S.F. Hassan, S.R. Wadia, Nucl. Phys. B526 (1998) 311.

[56] G. W. Gibbons and S.W. Hawking, Phys. Rev.D15 (1977) 2752.

[57] J.D. Bekenstein, Phys. Rev. D23 (1981) 287.

[58] D.N. Page Defining Entropy Bounds, hep-th/0007238.

[59] D.N. Page Subsystem Entropy Exceeding Bekenstein's Bound, hepth/0007237.

[60] D.N. Page Huge Violations of Bekenstein's Entropy Bound, gr$\mathrm{qc} / 0005111$.

[61] J.D. Bekenstein, On Page's examples challenging the entropy bound, grqc/0006003.

[62] D.N. Page, Phys. Rev. D26 (1982) 947.

[63] D.A. Lowe, Comments on a Covariant Entropy Conjecture, JHEP 9910 (1999) 026.

[64] W. Fischler and L. Susskind, Holography and Cosmology hepth/9806039.

[65] R. Bousso, JHEP 9907 (1999) 004.

[66] N. Kaloper and A. Linde, Phys. Rev. D60 (1999) 103509.

[67] E. Abdalla and L.A. Correa-Borbonet, Phys. Lett. B489 (2000) 383.

[68] N. Arkani-Hamed, S. Dimopoulos, N. Kaloper, J. March-Russell, Nucl. Phys. B567 (2000) 189.

[69] N. Arkani-Hamed, S. Dimopoulos, G. Dvali, Phys. Lett. B429 (1998) 263.

[70] J.C. Prince, in proc. Int. Symp. on Experimental Gravitational Physics, ed.P.F Michelson, Guangzhou, China(World Scientific, Singapore, 1988); J.C. Prince et al, NSF proposal 1996; A. Kapitulnik and T. Kenny, NSF proposal, 1997. 
[71] T. Kaluza, Sitzungsber. Preuss. Akad. Wiss. Berlin (Math.Phys.)(1921) K1:966.

[72] O. Klein, Z. Phys. 37 (1926) 895.

[73] D. Bailin and A. Love, Rept. Prog. Phys.50 (1987) 1087.

[74] J. M. Overduin and P. S. Wesson, Phys. Rept. 283 (1997) 303.

[75] A. Guth, Phys. Rev. D23 (1981) 347; K. Olive, Phys. Rept. 190 (1990) 307.

[76] G.F. Smoot et al, Astrophys. J. Lett 396(1992) L1.

[77] E. Verlinde, On the Holographic Principle in a Radiation Dominated Universe, hep-th/0008140.

[78] B. Wang, E. Abdalla and Ru-Keng Su, Phys.Lett. B503 (2001) 394. 